\title{
Thermal emission of spherical spinning ring particles
}

\section{The standard model}

\author{
C. Ferrari and C. Leyrat
}

Laboratoire AIM, Unité Mixte de Recherche No. 7158 CEA-CNRS-Université Paris 7, Denis Diderot, France
e-mail: cferrari@cea.fr, leyratc@discovery.saclay.cea.fr

Received 1 March 2005 / Accepted 20 September 2005

\section{ABSTRACT}

A simple new thermal model was developed to study the effects of the spherical shape of ring particles, of their finite thermal inertia, and of their spinning properties on the rings thermal emission. Ring particles are assumed to be scattered in a monolayer, a structure that might exist at least in the population of large particles embedded in Saturn's A and C rings. Their spin rates and obliquities are either identical or randomly distributed, but are constant with time. It is found that the infrared radiation from Saturn on spherical particles generates large azimuthal temperature asymmetries along the ring. Their amplitude and overall shape mainly depend on the viewing geometry, the spin properties, and the thermal inertia.

Key words. planets: rings - infrared: solar system - conduction - radiation mechanism: thermal

\section{Introduction}

According to numerical simulations of the collisional dynamics of Saturn's rings, meter-sized particles that represent most of the ring mass, are distributed in a few-particle-thick layer, while the smaller ones are scattered in a thicker multilayer (Brahic \& Sicardy 1981; Salo 1987a; Richardson 1994). The actual local vertical thickness and velocity dispersion in Saturn's rings can be derived from observations of bending waves.

The A ring vertical thickness is about a few-10-m (Shu 1982; Esposito et al. 1983), while the thinner C ring seems to be only about $1 \mathrm{~m}$-thick (Rosen 1989). The B ring is more difficult to probe due to its large optical depth. Recent ray-tracing simulations suggest that the tilt effect observed for Saturn's rings (Esposito et al. 1984) could be explained well by a very flat and dense ring inner layer surrounded by a halo of small particles (Salo \& Karjalainen 2003). A comparable tilt effect in thermal emission has been observed from the ground in the infrared domain. Thermal models that assume either a monolayer vertical structure (Froidevaux 1981) or a vertically heterogeneous multilayer (Kawata 1983) can both reproduce this effect quite well.

During collisions, random kinetic energy is transformed into rotational energy and the distribution of particle spins reaches an equilibrium. The ratio of rotational to kinetic energy is typically below 0.4 (Salo 1987b), depends heavily on either the friction parameters or on the surface irregularities of the particles (Salo 1987a). As the local velocity dispersion decreases significantly with this transfer, the local equilibrium of the ring disc is modified and the vertical thickness can be reduced by half as well (Salo 1987b). The spin rate for a single particle size in the inertial frame is nearly synchronous. Its mean components in the ring plane are zero, while its average vertical component $\omega_{z}$ is about a third of the mean motion. This mean value of the spin is constrained by the systematic velocity gradient inside the Keplerian ring disc. It varies only slightly with friction or ring optical thickness, in contrast to the dispersion in spin vectors (Salo 1987a; Araki 1991; Richardson 1994; Salo 1995). When particles of different sizes are present, simulations suggest that the smallest particles spin faster with a larger dispersion in spin rates because they are gravitationally scattered by the larger ones (Salo 1987a; Richardson 1994). Their spin can be five or ten times faster than the orbital motion. The distribution of spin obliquities is very broad for large particles: slightly retrograde in the particle rotating frame but prograde in the fixed inertial frame (Richardson 1994; Salo 1987b). Small particles are more randomly oriented and less clearly prograde (Salo 1987b).

The actual distributions of spin rates and orientations of Saturn's ring particles are unknown. Comparison of groundbased infrared observations with monolayer and multilayer thermal models have suggested slow rotators in all Saturn's main rings (Kawata 1983; Froidevaux 1981; Ferrari et al. 1999, 2005). Temperature measurements in the $\mathrm{C}$ ring by the Voyager IRIS spectrometer at high phase angle have shown that the particle emission function is highly anisotropic, also favoring the existence of slow rotators (Spilker et al. 2005). 
Ring particle surface temperatures are expected to depend heavily on their spin rate and orientation, just like temperatures on Earth vary with the hour of the day or the seasons. If its thermal inertia is small, a slowly rotating particle has, for most spin obliquities, a strong day-to-night thermal constrast compared to a fast rotating one. New spacecraft infrared observations of Saturn's rings in a near future should allow us to accurately measure such an anisotropy in the emission function, if any (Flasar et al. 2004).

Current Saturn's rings thermal models have not taken into account the thermal behavior of spherical particles with finite spin rate, variable obliquities, and finite thermal inertia. All but Aumman's model (Aumann \& Kieffer 1973) consider rings as a flat surface with either a multilayer (Kawata 1983) or a monolayer structure (Froidevaux \& Ingersoll 1980; Froidevaux 1981). The ring layers are made of particles with either high or low thermal day-to-night constrasts, i.e. a large or a small anisotropy in their emission function. The observed surface is lit at the same time by all the heating sources, i.e. the Sun, Saturn, and the nearby particles. All use ring or particle mean surface temperatures that result from an energy balance between absorbed and re-emitted fluxes. Only Aumman's model considers the finite thermal inertia of particles to study their transient thermal regime in the planetary shadow.

Our previous thermal model (Ferrari et al. 2005) considered a flat monolayer ring as proposed by Froidevaux (1981), and in addition included treatment of the transient thermal regime into the planetary shadow. Particles with either large or small anisotropy in their thermal emission were considered. This model was then compared with new mid-infrared ground-based images of Saturn's rings obtained with the CAMIRAS instrument mounted on the CHF telescope. The observed brightness temperatures constrained the particles to give them a very warm lit face or a highly anisotropic emission function. The fast heating of particles observed at the exit of the shadow showed that the thermal inertias of Saturn's B and C rings had to be as low as $5_{-2}^{+18} \mathrm{~J} \mathrm{~m}^{-2} \mathrm{~K}^{-1} \mathrm{~s}^{-1 / 2}$ and $6_{-4}^{+12} \mathrm{~J} \mathrm{~m}^{-2} \mathrm{~K}^{-1} \mathrm{~s}^{-1 / 2}$, respectively, i.e very low compared to the thermal inertia of solid water ice (Ferrari et al. 2005). Both results led to particles having to be slow rotators, slow enough for their lit face to be that warm. This model was unable to reproduce the azimuthal temperature variations detected in these same images, far away from the expected transient regime due to the crossing of the planetary shadow (Ferrari et al. 2005).

In this paper, we present a new model for the thermal emission of a monolayer ring. A ring of identical spherical particles with finite thermal inertia and parametrable spin rate and orientation is now considered. All particles are assumed to have constant obliquity and spin rate throughout their thermal history. Transient thermal phenomena during the particle thermal history are taken into account, such as the planetary shadow crossing or its diurnal cycle. The energy balance between incoming and outgoing fluxes is now treated at the scale of a particle elementary surface, i.e. a facet. This surface is usually not lit by all the heating sources at the same time, and its temperature differs from the nearby facets. The resulting anisotropy of the emission function of the particle can now be calculated for any spin rate and direction or thermal inertia. The particle mean temperature is replaced by the effective temperature of the observed face of the particle.

These simple but non-trivial assumptions are used in order to carefully study the effect of the rotational and thermal properties of particles on the observed ring temperatures. The model, its assumptions, and the equations that govern the thermal emission of the ring particles are described in Sect. 2. In Sect. 3, the diurnal and annual thermal cycles suffered by a particle are studied and discussed. Section 4 is devoted to the study of the role of model parameters on the effective temperature of a particle moving along its orbit and several examples are studied. They are far from being exhaustive, but they are simple enough to demonstrate the validity of model outputs.

\section{Model description}

In this standard model, ring particles absorb the visible sunlight, the visible and the infrared radiation from Saturn, and the visible and infrared radiation from their neighbors. The absorbed energy is stored as heat and later re-radiated into space. Inter-particle mutual shadowing reduces the incoming solar light. The lower the Sun is above the ring plane, the larger the mutual shadowing. Particles are periodically eclipsed into the planetary shadow. The length of the cooling episode into the shadow increases as sun sets on the rings.

The total absorbed flux and the direction of heating sources vary on a surface element of the spherical particle as it spins around its axis and orbits around the planet. Given the instantaneous directions of the sources, the model calculates the thermal map of a particle by solving the heat-transfer equation on each surface element, hereafter called a facet. As ring particles cannot yet be spatially resolved because of their size, the observer records the average spectrum emitted by the visible facets, from which an effective temperature is derived.

\subsection{Coordinate systems and rotations}

The position of the particle on its orbit is given by its distance $a$ and longitude $\phi_{\mathrm{R}}$ in the planet reference frame $\mathrm{P}\left(X_{\mathrm{P}}, Y_{\mathrm{P}}, Z_{\mathrm{P}}\right)$ (Fig. 1). By convention in ring thermal studies, the ring longitude $\phi_{\mathrm{R}}=0$ at the anti-solar direction and increases in the direction of Keplerian motion. The particle follows a circular orbit with mean motion $\Omega(a)$. The observer's and Sun's spherical coordinates in this frame are $\left(r_{\mathrm{SC}}, B, \phi_{\mathrm{SC}}\right)$ and $\left(D_{\mathrm{UA}}, B^{\prime}, \phi_{0}=\right.$ $\left.180^{\circ}\right)$, respectively. The $\mathrm{R}$-frame $\left(X_{\mathrm{R}}, Y_{\mathrm{R}}, Z_{\mathrm{R}}\right)$ is centered on the particle and rotates at constant angular velocity $\Omega$ around the planet. The $Y_{\mathrm{R}}$-axis is locked to the solar direction and $Z_{\mathrm{R}}$-axis towards the orbit's North pole. Any facet on the particle surface is located by its spherical coordinates $(r, \delta, \phi)_{\mathrm{L}}$ in the local L-frame $\left(X_{\mathrm{L}}, Y_{\mathrm{L}}, Z_{\mathrm{L}}\right)$ centered on the particle (Fig. 2). The particle surface is divided into 288 facets of $15 \times 15^{\circ 2}$. The size of the facet was chosen as a compromise between calculation duration and accuracy. The relative difference in effective temperature between a surface sampled every $15^{\circ}$ or every $5^{\circ}$ is $\sim 0.1 \%$ only on average. The L-frame rotates in the R-frame along the spin axis oriented along $Z_{\mathrm{L}}$. The spin orientation is defined at the beginning of the thermal history by its right ascension and declination $\left(\alpha_{\mathrm{P}}, \delta_{\mathrm{P}}\right)$ in the R-frame. At initial time, 


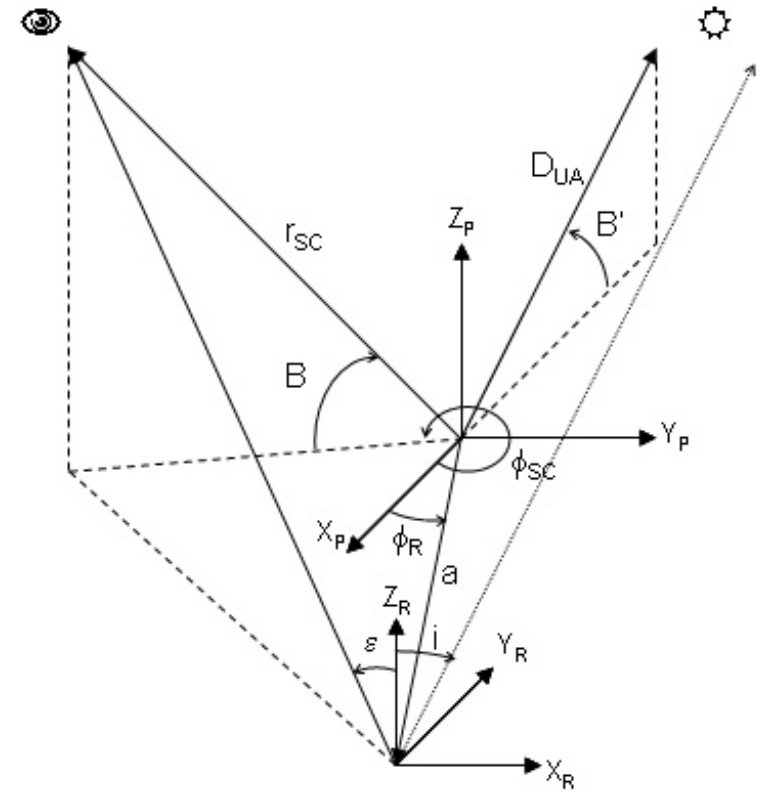

Fig. 1. Schematic diagram of the model geometry displaying the planet reference frame $\mathrm{P}\left(X_{\mathrm{P}}, Y_{\mathrm{P}}, Z_{\mathrm{P}}\right)$ and the $\mathrm{R}$-frame $\left(X_{\mathrm{R}}, Y_{\mathrm{R}}, Z_{\mathrm{R}}\right)$, rotating with the particle at distance a from the planet center and angular velocity $\Omega$. The $Z_{\mathrm{P}}$-axis is parallel to the planet's North pole direction and the $X_{\mathrm{P}}$-axis defines the origin of longitudes in the equatorial plane in the antisolar direction. The $Z_{\mathrm{R}}$-axis is parallel to the $Z_{\mathrm{P}}$-axis, and the $Y_{\mathrm{R}}$-axis is locked to the solar direction.

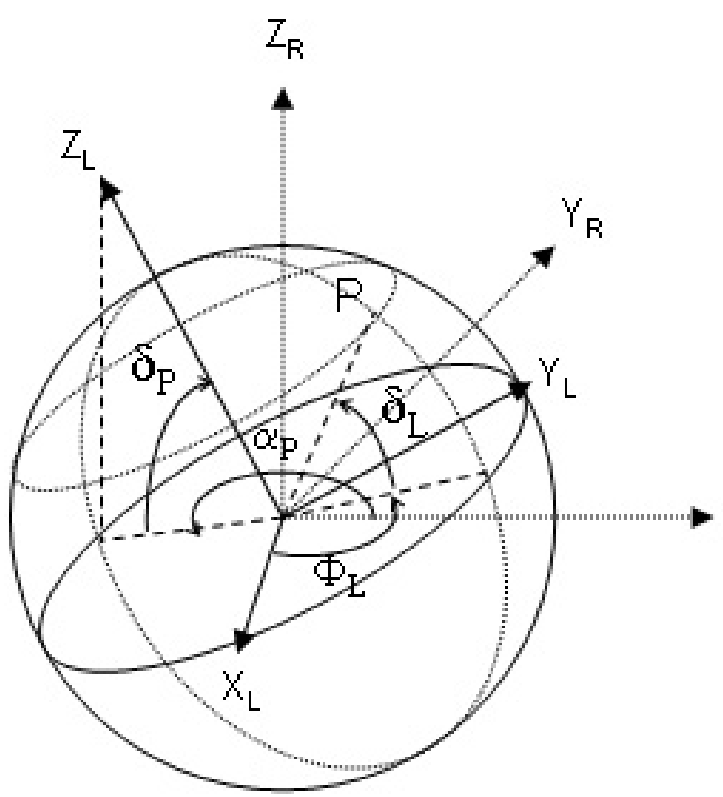

Fig. 2. Schematic diagram of the local particle's reference frame L $\left(X_{\mathrm{L}}, Y_{\mathrm{L}}, Z_{\mathrm{L}}\right)$, rotating at constant spin rate $\omega$ in the $\mathrm{R}$-frame $\left(X_{\mathrm{R}}, Y_{\mathrm{R}}, Z_{\mathrm{R}}\right)$. The $Z_{\mathrm{L}}$-axis is oriented along the particle spin axis and is defined by its right ascension $\alpha_{\mathrm{P}}$ and declination $\delta_{\mathrm{P}}$ relative to the R-frame.

$X_{\mathrm{L}}$ and $X_{\mathrm{R}}$ axes are parallel. The local longitude $\Phi_{\mathrm{L}}$ is relative to the $X_{\mathrm{L}}$-axis and increases in the direct direction. In the standard model, the spin axis is not precessing in the R-frame. It can be pointed to any direction. Particle spin rate $\omega$ in the inertial frame can take values between $0.1 \Omega$ and $10 \Omega$, by step of $0.1 \Omega$, both in the prograde and retrograde directions.

The thermal history of a particle is calculated over a pe$\operatorname{riod} T_{\mathrm{H}}$ that is commensurate to the particle day $T_{\mathrm{d}}=2 \pi / \omega$ and the particle orbital period $T_{\mathrm{a}}=2 \pi / \Omega$, i.e. over a ten orbitperiod $T_{\mathrm{H}}=10 T_{\mathrm{a}}=10 T_{\mathrm{d}} \omega / \Omega$. For some values of $\omega / \Omega$ (multiples of 0.2 or 0.5 ), it can be shortened to 5 or $2 T_{\mathrm{a}}$. If $\omega / \Omega<1$, every orbit is sampled in azimuth every 5 degrees in order to keep calculation time reasonable. It corresponds to a sampling time interval of $t_{\mathrm{S}}=T_{\mathrm{a}} / n t$, where $n t=72$. In the case of faster rotators $(\omega / \Omega>1)$, the day period $T_{\mathrm{d}}$ is sampled every $T_{\mathrm{d}} / n t$ seconds where $n t=40$ or 20 , depending on the ratio $\omega / \Omega$ to limit the calculation time. The thermal history period $T_{\mathrm{H}}$ is shortened to one orbital period $T_{\mathrm{a}}$ when $\omega / \Omega$ is an integer.

\subsection{Effective temperature and ring brightness temperature}

The infrared spectrum $I_{\mathrm{P} v}$ of a ring particle at ring longitude $\phi_{\mathrm{R}}$ is the average brightness of its visible hemisphere. It can be written as:

$I_{\mathrm{P} v}\left(\phi_{\mathrm{R}}\right)=\frac{\iint \epsilon_{v, \phi, \delta} B_{v}\left(T_{S}(\phi, \delta)\right) \cos \theta_{V} \mathrm{~d} S_{\phi, \delta}}{\pi r^{2}}$

where $\theta_{V}$ is the local emission angle at azimuth $\phi$ and latitude $\delta$ in the local L-frame. Here $S_{\phi, \delta}$ represents the local emitting surface of the facet, $T_{\mathrm{S}}(\phi, \delta)$ its temperature, $\epsilon_{v, \phi, \delta}$ its emissivity as a function of frequency $v$, and $r$ the particle radius. In this study, the emissivity is supposed to be uniform and constant, i.e. $\epsilon_{v, \phi, \delta}=\epsilon$. If $\cos \left(\theta_{V}\right)<0$, the facet is invisible. The quantity $I_{\mathrm{P} v}$ differs from a blackbody spectrum as the particle is generally not isothermal. The particle effective temperature $T_{\mathrm{EFF}}$ is deduced from the spectrum with Stefan's law:

$\int I_{\mathrm{P} v}\left(\phi_{\mathrm{R}}\right) \mathrm{d} v=\epsilon \sigma T_{\mathrm{EFF}}^{4}\left(\phi_{\mathrm{R}}\right)$.

After the particles' effective temperature, the brightness temperature of the ring will depend on their filling factor in the ring plane and on how they are hiding each other relative to the observer. Particles are distributed in a monolayer disc of geometric optical depth $\tau$, defined as the fractional area occupied by particles as seen by an observer at elevation $B=90^{\circ}$ above the ring plane. When the elevation decreases, this fraction increases as $\tau / \mu$ where $\mu=\sin (B)$. Below some elevation threshold, particles are hiding each other. Only a fraction $\mathrm{C}(\tau, B)$ of their surface is visible, and it is emissive from the observer view point. It decreases with increasing optical depth for a given elevation as screening between particles increases. The non-shadowing/screening coefficient $\mathrm{C}\left(\tau, B^{\prime}\right.$ or $\left.B\right)$ can be expressed either analytically in very specific configurations or experimentally from a random distribution of spheres distributed in a monolayer (Froidevaux 1981). The emissive fraction of the observed surface is, therefore, $F F=C(\tau, B) \tau / \mu$. The observed spectrum $I_{v}$ of the ring monolayer can be expressed as a function of the ring particle spectrum $I_{P v}$ at frequency $v$ as:

$I_{v}\left(\phi_{\mathrm{R}}\right)=I_{\mathrm{P} v}\left(\phi_{\mathrm{R}}\right) C(\tau, B) \tau / \mu \leq I_{\mathrm{P} v}\left(\phi_{\mathrm{R}}\right)$ 


\subsection{Equations}

Illuminating sources. Heating sources are the Sun, the lit hemisphere and infrared emission of the central planet, the sunlight scattered by close nearby particles and their infrared emission. The absorbed flux $F_{\mathrm{T}}$ at each point on the surface of a particle located at ring longitude $\phi_{\mathrm{R}}$ is written:

$$
\begin{aligned}
\left(1-A_{\mathrm{V}}\right) & \frac{S\left(\phi_{\mathrm{R}}\right)}{D_{\mathrm{AU}}^{2}} C\left(\tau, B^{\prime}\right) \cos \left(\theta_{0 \mathrm{~L}}\right) \\
& +A_{\mathrm{V}}\left(1-A_{\mathrm{V}}\right) \frac{S\left(\phi_{\mathrm{R}}\right)}{D_{\mathrm{AU}}^{2}} C\left(\tau, B^{\prime}\right) \frac{\Omega_{\mathrm{R}}}{4 \pi} \\
& +\int \frac{\sigma T_{\mathrm{P}}^{4}}{\pi} \cos \left(\theta_{S}\right) \frac{\mathrm{d} S_{\mathrm{SAT}}}{l_{S}^{2}} \\
& +A_{S}\left(1-A_{\mathrm{V}}\right) \frac{S}{\pi D_{\mathrm{AU}}^{2}} \int \cos \left(\theta_{S}\right) \cos \left(\theta_{0 S}\right) \frac{\mathrm{d} S_{\mathrm{SAT}}}{l_{\mathrm{S}}^{2}} \\
& +F_{\mathrm{T}} \frac{\Omega_{\mathrm{R}}}{4 \pi}=F_{\mathrm{T}}=\epsilon \sigma T_{S}^{4} .
\end{aligned}
$$

This equation originates from the monolayer formalism developed by Froidevaux (1981). The variables are the same here and in our previous model (Ferrari et al. 2005), but are applied at the scale of the particle facet. The first term of Eq. (4) represents the absorbed solar flux coming from local incident angle $\theta_{0 \mathrm{~L}}$, with consideration of the non-shadowing factor. The second term corresponds to the sunlight scattered by nearby particles subtending a solid angle $\Omega_{\mathrm{R}}$ and subsequently absorbed. It is assumed to be isotropic. The third term is the Saturn infrared input. For each facet, the flux is integrated over all the Saturn surface elements with apparent surface $\mathrm{d} S_{\mathrm{SAT}}$, incoming local direction $\theta_{S}$, and distance $l_{S}$ to the facet. In the case of Saturn's rings, this angle varies from $2.09 \mathrm{sr}$ to $0.6 \mathrm{sr}$ between the inner edge of the $\mathrm{C}$ ring $(a=74000 \mathrm{~km})$ and the outer edge of the A ring $(a=136900 \mathrm{~km})$. Partial obscuration of this solid angle by nearby particles is neglected for low geometric optical depths $\tau$. For $\tau>0.7$, particles are too packed to stay in a monolayer. They cannot see both Saturn hemispheres. In this case the incoming flux is divided by 2 . This is to be distinguished from the photometric optical depth $\tau_{\mathrm{P}}$ which gives a non zero probability $\mathrm{e}^{-\tau_{\mathrm{P}}}$ of a photon passing through the ring when $\tau_{\mathrm{P}}>0.7$. The fourth term is the sunlight reflected by Saturn onto the particle and absorbed, and $\theta_{0 S}$ is the solar incident angle on the Saturn surface element $\mathrm{d} S_{\mathrm{SAT}}$. If $\cos \left(\theta_{0 \mathrm{~L}}\right)<0$, the facet is unlit, if $\cos \left(\theta_{S}\right)<0$, the surface element on Saturn does not light the facet. It is not illuminated if $\cos \left(\theta_{0 S}\right)<0$. The fifth term, representing the infrared flux from nearby particles, is assumed to be isotropic. The infrared radiation is supposed to be fully absorbed.

The non-shadowing factor $C\left(\tau, B^{\prime}\right)$ is assumed to be the same for every facet of a particle in a ring of geometric optical depth $\tau$ illuminated by the Sun at elevation $B^{\prime}$. This is, of course, not true as facets at high latitudes of a particle are most probably receiving more sunlight than equatorial ones, if they are pointing to the lit face of the ring. It might be possible to use our formalism to calculate a specific non-shadowing factor for a facet, given its coordinates, the spin rate and orientation, the geometric optical depth, and the particle longitude at any time. But this would increase the computation time by a lot.
Table 1. Definitions of variables.

\begin{tabular}{cl}
\hline \hline$A_{\mathrm{V}}$ & Ring particle bolometric Bond albedo \\
$A_{S}$ & Saturn bolometric Bond albedo $=0.342$ \\
$B$ & Earth elevation above the ring plane \\
$B^{\prime}$ & Solar elevation above the ring plane \\
$C_{\mathrm{H}}$ & Specific heat $\left(\mathrm{J} \mathrm{kg}^{-1} \mathrm{~K}^{-1}\right)$ \\
$C\left(\tau, B^{\prime}\right)$ & Fractional lit area of the particle \\
$C(\tau, B)$ & Fractional visible area of the particle \\
$D_{\mathrm{AU}}$ & Sun-Saturn distance in astronomical units \\
$\epsilon$ & Infrared emissivity of ring particles, $\epsilon=1$ \\
$\Gamma$ & Thermal inertia $\left(\mathrm{J} \mathrm{m}^{-2} \mathrm{~K}^{-1} \mathrm{~s}^{-1 / 2}\right)$ \\
$K$ & Thermal conductivity $\left(\mathrm{W} \mathrm{m}^{-1} \mathrm{~K}^{-1}\right)$ \\
$\Omega_{\mathrm{R}}$ & Solid angle subtended by all nearby particles \\
$\omega$ & Particle spin rate \\
$\Omega$ & Particle mean motion \\
$r$ & Radius inside particle \\
$\rho$ & Density of the particle $\left(\mathrm{kg} \mathrm{m}^{-3}\right)$ \\
$S, \sigma$ & Solar constant $=1370 \mathrm{~W} \mathrm{~m}^{-2}$, Stefan constant \\
$\tau$ & Ring geometric optical depth \\
$T_{\mathrm{EFF}}$ & Effective temperature of the visible surface \\
$T_{S}$ & Surface temperature of a particle facet \\
$T_{\mathrm{P}}$ & Saturn planet effective temperature, $T_{\mathrm{P}}=95 \mathrm{~K}$ \\
\hline
\end{tabular}

To get a realistic description of the heating contribution of the neighboring particles on the current test particle facet is complex. In a first approximation, the temperature of the neighbors is assumed to be equal to the facet temperature, which is not true if particles are not isothermal. As neighbors are heated up by the sunlight, their infrared contribution increases. In the planetary shadow, only their infrared contribution is present, and it decreases with time as they are cooling. Their visible contribution is at its maximum when the current facet is not facing the Sun, therefore facing their lit faces. It disappears when the facet is lit by the Sun. If particles are regularly distributed in the ring plane, the solid angle subtended by the eight closest neighbors is $\Omega_{\mathrm{R}}=6\left(1-\mathrm{e}^{-\tau}\right)$ (Ferrari et al. 2005). But these eight neighbors are not seen at the same time. Most probably only two are visible at any given time; therefore $\Omega_{\mathrm{R}}=1.5\left(1-\mathrm{e}^{-\tau}\right)$.

Heat conduction and thermal properties. Saturn's ring particles are cm-to-meter-sized bodies, primarily covered with water ice (French \& Nicholson 2000; Pilcher et al. 1970). Their surface structure is almost unknown, but spectroscopic observations suggest that they are covered with a regolith of dust grains, from a few microns to submillimeter in size (Clark \& Mc Cord 1980; Poulet \& Cuzzi 2002). Their thermal inertia is very low, on the order of a few $\mathrm{J} \mathrm{m}^{-2} \mathrm{~K}^{-1} \mathrm{~s}^{-1 / 2}$, assuming heat transfer by conduction only. The regolith might be either compact and fractured by cracks or highly porous (Ferrari et al. 2005).

The treatment of heat diffusion here is exactly the same as in our previous model (Ferrari et al. 2005) but it applies to any facet of the spherical particle. For simplification, only heat transfer through depth is accounted for, which is reasonable as the planar dimensions of the facet are much larger than the 
thermal skin depths of the system. The transfer is controlled by the heat diffusion equation at radius $r$ into the particle:

$\frac{1}{r^{2}} \frac{\partial}{\partial r}\left(r^{2} \frac{\partial T}{\partial r}\right)=\frac{\rho C_{\mathrm{H}}}{K} \frac{\partial T}{\partial t}$

assuming axisymmetry of the problem in spherical coordinates, homogeneity and isotropy of thermal properties. At the center of the particle, the heat flow is supposed to be zero. The boundary conduction at the surface of the particle is:

$F_{T}-\epsilon \sigma T_{S}^{4}\left(1-\frac{\Omega_{R}}{4 \pi}\right)=K \frac{\partial T}{\partial r} ;$

i.e. the heat flow at the surface is equal to the difference between input flux $F_{T}$ and flux radiated by the surface. The facet temperature $T_{S}\left(\phi_{\mathrm{R}}\right)$ is recalculated until the average emitted flux over an orbital period equals the average absorbed flux. For very low thermal conductivity, i.e. low diffusivity and small thermal skin depth, $T_{S}\left(\phi_{\mathrm{R}}\right)$ should look like the instantaneous temperature $T_{\mathrm{I}}\left(\phi_{\mathrm{R}}\right)=\left(\frac{F_{T}\left(\phi_{\mathrm{R}}\right)}{\epsilon \sigma}\right)^{1 / 4}$. For very high thermal conductivity, the material only reacts slowly to flux variations. In this case, the facet temperature tends to be constant, about the average temperature $T_{\mathrm{M}}(\theta)=\left(\frac{F_{\mathrm{M}}}{\epsilon \sigma}\right)^{1 / 4}$ where $F_{\mathrm{M}}$ is the average incident flux.

Transfer of energy by any other means than conduction is neglected here. As a consequence, the whole incident radiation on the surface is assumed to be deposited in the first micrometers of the regolith. The energy might in fact be accumulated at different depths depending on the material composition, the porosity, and the wavelength considered. The electric skin depth $\delta_{\mathrm{E}}$ of solid water ice frost is on the order of $10 \mu \mathrm{m}$ between 7 and $70 \mu \mathrm{m}$ wavelengths, and it increases gradually to $1 \mathrm{~mm}$ at $1000 \mu \mathrm{m}$. This means that part of the incident energy can reach deep layers into the regolith at large wavelengths, and even deeper if the ice regolith is porous. Also some of the observed infrared flux can come from deep layers.

The thermal inertia $\Gamma=(1-p) \sqrt{K \rho C_{\mathrm{H}}}$ is the physical property that controls both the temperature variations at the surface and the capacity of the subsurface to store energy, given its porosity $p$. We consider here that $\Gamma$ depends mainly on the thermal conductivity $K$, as density $\rho$ and specific heat capacity $C_{\mathrm{H}}$ are relatively less variable with the nature of the material. Thus $K$ will be considered as a primary variable of our model instead of $\Gamma$. The code is stable in a continuous range of $K$ values between a few $10^{-6}$ to $1 \mathrm{~W} \mathrm{~m}^{-1} \mathrm{~K}^{-1}$. This thermal system has two thermal skin depths, the diurnal $l_{\omega}=\sqrt{\alpha / \omega}$ and the orbital (annual) one, $l_{\Omega}=\sqrt{\alpha / \Omega}$, where $\alpha=K / \rho C_{\mathrm{H}}$ is the diffusivity of the surface material. The calculation has to be conducted at several times the largest skin depth, and sampling has to be sufficient to describe temperature variations in depth at the scale of the smallest one. For thermal inertia $\Gamma \sim 5 \mathrm{~J} \mathrm{~m}^{-2} \mathrm{~K}^{-1} \mathrm{~s}^{-1 / 2}$, specific heat capacity $C_{\mathrm{H}}(T)=7.49 T+90 \sim 800 \mathrm{~J} \mathrm{~kg}^{-1} \mathrm{~K}^{-1}$ (Klinger 1981) at ring temperatures, and density $\rho=918 \mathrm{~kg} \mathrm{~m}^{-3}$, the thermal conductivity $K$ is $\sim 4 \times 10^{-5} \mathrm{~W} \mathrm{~m}^{-1} \mathrm{~K}^{-1}$ for a porosity $p=0$. For a large porosity, $p=0.9, \rho=91,8 \mathrm{~kg} \mathrm{~m}^{-3}$ and $K \sim 4 \times$ $10^{-3} \mathrm{~W} \mathrm{~m}^{-1} \mathrm{~K}^{-1}$ (Ferrari et al. 2005). The annual skin depth $l_{\Omega}$ ranges between 0.5 -to- $0.7 \mathrm{~mm}$ from the inner $\mathrm{C}$ ring to outer
A ring as the diffusivity is $\alpha \sim 5 \times 10^{-11} \mathrm{~m}^{2} \mathrm{~s}^{-1}$. The diurnal skin depth $l_{\omega}=l_{\Omega} \sqrt{\Omega / \omega}$, i.e. between $0.2 \mathrm{~mm}$ and $2 \mathrm{~mm}$ on average between the $C$ and A rings, given that spin rates $\omega$ considered here range from $0.1 \Omega$ to $10 \Omega$. The thermal skin depth is smaller than the electrical skin depth in the wavelength domain from $200 \mu \mathrm{m}$ to $1000 \mu \mathrm{m}$. The diffusion equation used here is, therefore, no longer valid in this particular wavelength domain. The smallest particles in Saturn's rings are much larger than these thermal skin depths. For this reason, particles of all sizes in Saturn's rings will exhibit the same thermal behavior if they have the same thermal properties and spin. The impact of the particle size on the observed temperature is therefore not studied here. Particles of different sizes will exhibit different thermal histories because their dynamical histories are different.

\section{Ring particle temperatures}

The model is validated by checking its behavior against expected tendencies or values in limiting cases. Simulations follow here to study the diurnal and annual surface temperatures $T_{S}$ of some typical facets of the particle surface. We examine the influence of the thermal conductivity, the heating sources, the planetary eclipse, or of the spin rate $\omega$.

\subsection{Diurnal and annual thermal cycles}

The thermal history of a particle facet is first illustrated in the simple case where the particle is a single isolated synchronous satellite lit only by the Sun (Fig. 3). The facet is at local coordinates $(\phi, \delta)_{\mathrm{L}}=\left(90^{\circ}, 0^{\circ}\right)$, i.e. in the equatorial plane of the particle facing Sun at initial time $\left(\phi_{\mathrm{R}}=180^{\circ}\right)$. Here the diurnal cycle also corresponds to the annual cycle. For very low thermal conductivity, the maximum temperature is observed at the sub-solar point $\left(\phi_{\mathrm{R}}=180^{\circ}\right)$ and is almost equal to the expected sub-solar temperature $T_{\mathrm{SS}}=\left(S\left(1-A_{\mathrm{V}}\right) / \epsilon \sigma D_{\mathrm{UA}}^{2}\right)^{1 / 4}=109 \mathrm{~K}$ for $D_{\mathrm{UA}}=9.25 \mathrm{AU}, A_{\mathrm{V}}=0.5$ and $\epsilon=1$. The day-to-night temperature contrast decreases with increasing thermal conductivity, and the maximum temperature is always reached later in the afternoon, as expected. For very high thermal conductivity, the surface temperature is constant throughout the day. It is determined by the average flux received $F_{\mathrm{M}}$ on the facet throughout the day, $2.54 \mathrm{~W} \mathrm{~m}^{-2}$ here, and the expected value is $T_{\mathrm{M}}=\left(F_{\mathrm{M}} / \epsilon \sigma\right)^{1 / 4}=81.9 \mathrm{~K}$, actually given by the model. It is also verified that the sub-solar temperature decreases with $\left(\cos \delta_{\mathrm{L}}\right)^{1 / 4}$ as the incident flux decreases by this same amount northward or southward. This facet of the synchronous particle will never receive infrared or visible heating flux from the planet, as it is located on the far side as viewed from Saturn.

A facet on nearly the opposite side of the particle at local coordinates $(\phi, \delta)_{\mathrm{L}}=\left(-75^{\circ}, 0^{\circ}\right)$ for example, will always face the planet (Fig. 4). This facet receives full sunlight near $\phi_{\mathrm{R}}=0^{\circ}$. The absorption of Saturn's infrared emission provides about $2.4 \mathrm{~W} \mathrm{~m}^{-2}$ at a slight incidence angle $\left(15^{\circ}\right)$ at the distance of $a=84000 \mathrm{~km}$. The temperature is increased by $19 \mathrm{~K}$ at $\phi_{\mathrm{R}}=180^{\circ}$ and about $13 \mathrm{~K}$ at $\phi_{\mathrm{R}}=0^{\circ}$, assuming a thermal conductivity $K=0.0001 \mathrm{~W} \mathrm{~m}^{-1} \mathrm{~K}^{-1}$. The contribution from 


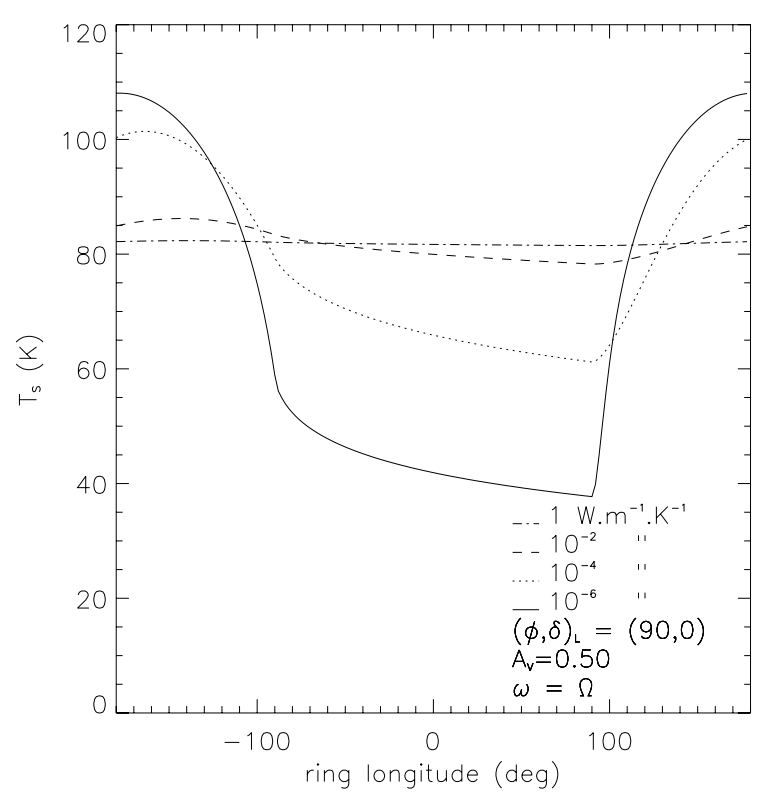

Fig. 3. Diurnal cycle of a particle pacet at local coordinates $(\phi, \delta)_{\mathrm{L}}=$ $\left(90^{\circ}, 0^{\circ}\right)$ as a function of thermal conductivity $\mathrm{K}$ of the material $\left(\mathrm{W} \mathrm{m}^{-1} \mathrm{~K}^{-1}\right)$. The spin axis is perpendicular to the ring plane, the particle is prograde, and $\omega=\Omega(a=84000 \mathrm{~km})$. The Sun coordinates in the planet frame are $\left(D_{\mathrm{UA}}=9.25 \mathrm{AU}, B^{\prime}=0^{\circ}, \phi_{0}=180^{\circ}\right)$. It is the only heating source. The planetary shadow episode and mutual inter-particle shadowing are not taken into account. The temperature is calculated every $5^{\circ}$ in ring longitude. This facet is always facing away from the planet.

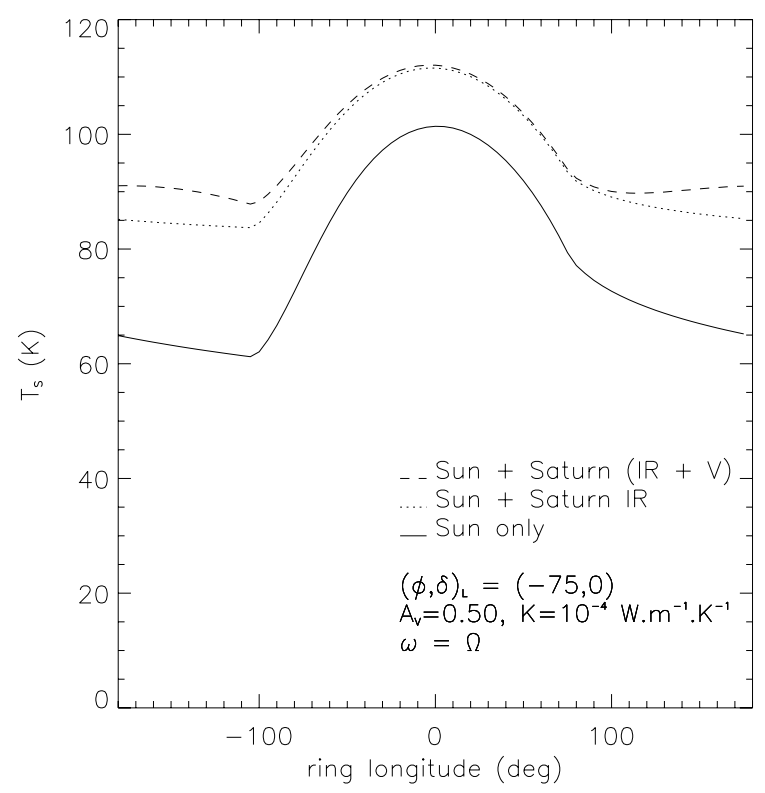

Fig. 4. Diurnal cycle of a particle facet at local coordinates $(\phi, \delta)_{\mathrm{L}}=$ $\left(-75^{\circ}, 0^{\circ}\right)$ as a function of incoming heating fluxes: Sun alone, or with the infrared emission from Saturn (dotted line) or with both visible and infrared emission from the planet (dashed line). The other parameters are identical to those of Fig. 3.

the visible Saturn hemisphere adds $0.7 \mathrm{~W} \mathrm{~m}^{-2}$ of heating flux at $\phi_{\mathrm{R}}=180^{\circ}$ and nothing at $\phi_{\mathrm{R}}=0^{\circ}$, as this hemisphere is invisible from the facet at this longitude. The maximum

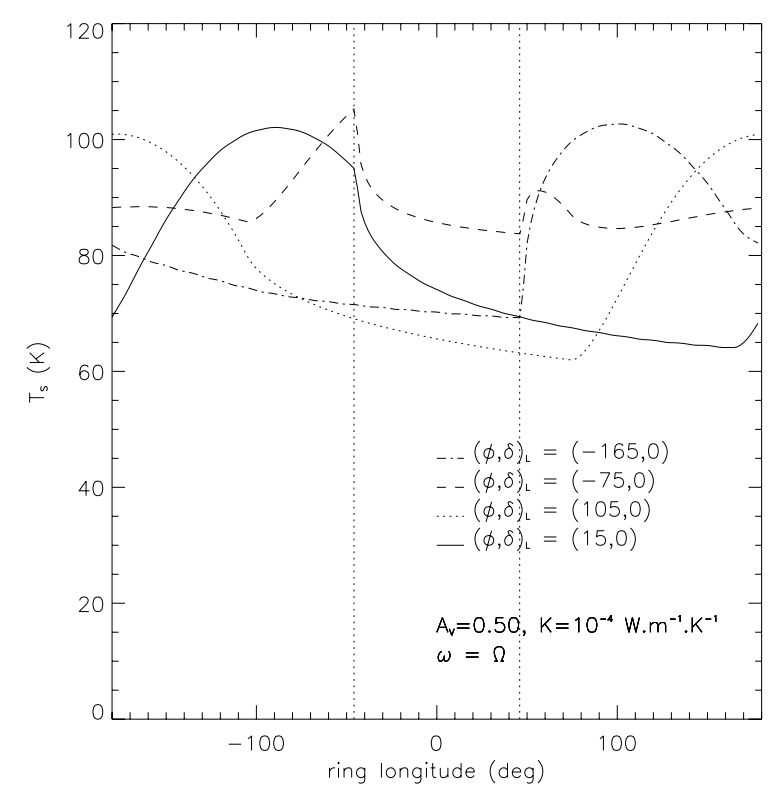

Fig. 5. Diurnal cycles of four different facets of a ring particle at local coordinates $(\phi, \delta)_{\mathrm{L}}$ when the eclipse into the planetary shadow is considered. The particle is eclipsed by Saturn in the ring longitude range $\left[-45.9^{\circ}, 45.9^{\circ}\right]$. Spin properties, particle and Sun coordinates are similar to those of Fig. 3. The heating fluxes from the Sun and Saturn's infrared and visible hemispheres are taken into account. The mutual inter-particle shadowing is not included.

temperature offset due to the visible hemisphere is about $6 \mathrm{~K}$ at $\phi_{\mathrm{R}}=0^{\circ}$ at this distance of the planet.

In practice, ring particles are eclipsed into the planetary shadow every orbit. This eclipse significantly disturbs the diurnal and annual thermal cycles of facets, especially if the thermal inertia is low. Figure 5 shows that for a facet of a synchronous particle on the hemisphere facing the planet at anytime, for example located at $(\phi, \delta)_{\mathrm{L}}=\left(-75^{\circ}, 0^{\circ}\right)$, the diurnal cycle is strongly perturbed by the eclipse around $\phi_{R}=0^{\circ}$. The farthest side of the particle relative to Saturn, at coordinates $(\phi, \delta)_{\mathrm{L}}=\left(105^{\circ}, 0^{\circ}\right)$ for example, is not perturbed as it is facing away from the sun when the particle crosses the shadow. The diurnal cycle of the other two facets are only slightly perturbed.

More generally, for particles with a non-integer $\omega / \Omega$ ratio, a full thermal cycle is accomplished after 10 revolutions for our chosen steps for spins of $0.1 \omega / \Omega$. For a spin $\omega=6.3 \Omega$, 6.3 rotations happen in one revolution, and the eclipse into the planetary shadow modifies the diurnalcycle at different epochs (Fig. 6a). Each bright peak corresponds to the noon time on the facet, about every $60^{\circ}$ in ring longitude. The facet is lit by Saturn about half a spin period later, i.e. about $30^{\circ}$ later on the orbit, which explains the existence of secondary bumps. The diurnal temperature gradients never exceed $25 \mathrm{~K}$ for the given thermal conductivity. For a spin of $\omega=0.3 \Omega$, only 3 rotations take place in 10 revolutions (Fig. 6b). Every three revolutions, the surface temperature behaves similarly, like for example in revolutions $1,4,7$, and 10 . Diurnal temperature gradients may exceed $30 \mathrm{~K}$ some days. In these intermediate cases, the facet has very different temperatures at a given orbital longitude depending on the phase of the thermal cycle. At a given ring longitude, every phase can be observed with equal probability. 

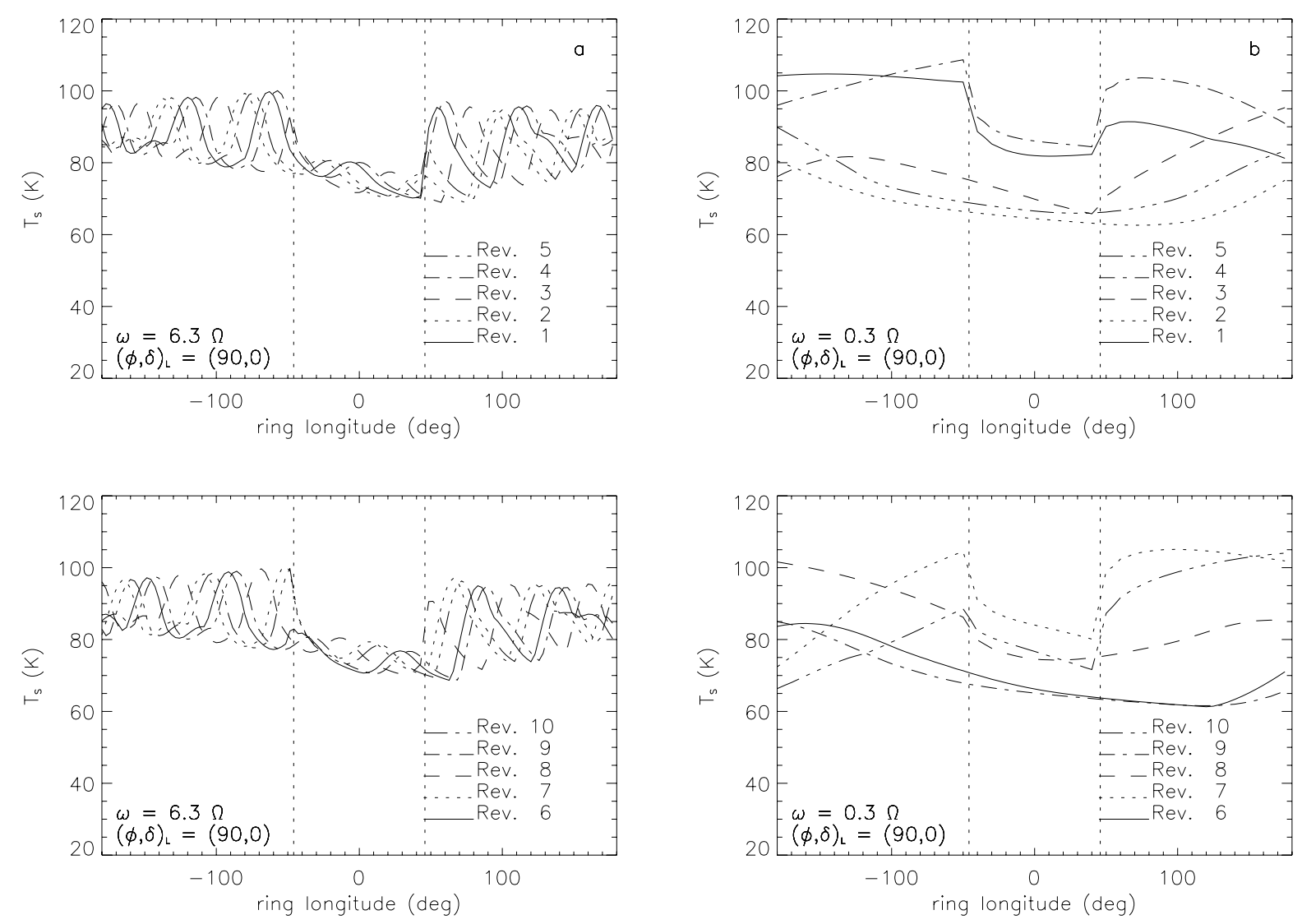

Fig. 6. Annual thermal cycles of the particle facet located at local coordinates $(\phi, \delta)_{\mathrm{L}}=\left(90^{\circ}, 0^{\circ}\right)$. Fluxes from the Sun and Saturn's infrared and visible hemispheres are taken into account. The particle is eclipsed by Saturn in the ring longitude range $\left[-45.9^{\circ}, 45.9^{\circ}\right]$. Its distance to Saturn is $a=84000 \mathrm{~km}$, its thermal conductivity $K=10^{-4} \mathrm{~W} \mathrm{~m}^{-1} \mathrm{~K}^{-1}$, and its Bond albedo $A_{\mathrm{V}}=0.5$. The spin axis is perpendicular to the ring plane, and the rotation is prograde. a) Particle spin is $\omega=6.3 \Omega$. The particle follows a full thermal cycle after 10 orbital revolutions (Rev. 1 to 10) or 63 rotations. b) Particle spin is $\omega=0.3 \Omega$. The particle follows a full thermal cycle after 10 orbital revolutions (Rev. 1 to 10) or 3 rotations.

The final azimuthal profile of the particle's effective temperature is obtained by averaging fluxes from the visible facets seen at the different phases of their thermal cycle.

The contribution of nearby particles to the particle heating was calculated for a facet located at local coordinates $\left(-75^{\circ}, 0^{\circ}\right)$ in the same conditions as used for Fig. 4 but with the shadow eclipse taken into account (Fig. 7). Due to this cooling event, the average surface temperature is then comparatively cooler. As described earlier, the visible contribution of the neighbors disappears when the facet is lit by the Sun, and their infrared contribution increases as long as they are also lit. For low optical depth $(\tau=0.1)$, the total absorbed flux from the neighbors is about $0.1 \mathrm{~W} \mathrm{~m}^{-2}$, i.e. only one percent of the solar contribution (Fig. 7a). For a larger optical depth $(\tau=0.5)$, it reaches about $0.4 \mathrm{~W} \mathrm{~m}^{-2}$ on average. The impact on the facet temperature is negligible for $\tau=0.1$, but the temperature can increase by 1 or $2 \mathrm{~K}$ when $\tau=0.5$ (Fig. 7b). The visible contribution decreases with the Bond albedo, in contrast to the infrared contribution.

\subsection{Effective temperature of a ring particle}

The observed specific intensity of the particle $I_{\mathrm{P} v}$ will not behave like a blackbody curve because of the patchwork of facet temperatures. Most of the time, $\mathrm{B}\left(T_{\mathrm{EFF}}\right)$ is smaller than $I_{\mathrm{P} v}$ at large wavenumbers, as $T_{\mathrm{EFF}}$ is lower than the hottest visible facets on the particle, and it is larger than $I_{\mathrm{P} v}$ at small wavenumbers as $T_{\mathrm{EFF}}$ is larger than the minimum temperatures of these same facets. Figure 8 displays the residual $\left(I_{\mathrm{P} v}-B\left(T_{\mathrm{EFF}}\right)\right) / I_{\mathrm{P} v}$ averaged over the ring longitude $\phi_{\mathrm{R}}$ for a synchronous prograde particle. The particle is only lit by the Sun and suffers no eclipse into the shadow. As the thermal conductivity rises, the particle tends to have a smaller day/night contrast in the azimuth direction $\phi_{\mathrm{L}}$ (for a spin direction perpendicular to the ring plane), but it keeps a thermal gradient in the latitudinal direction $\delta_{\mathrm{L}}$ due to variable incidence of the main heating sources. The residuals tend to decrease, as expected, but stay non-zero because the particle is not isothermal in the latitudinal direction. They equal zero for perfectly isothermal particles.

\section{Azimuthal variations in effective temperature}

We show here that large azimuthal variations of the effective temperature are created because of the position of the planet at the center of the particle orbit and the 3D volume of the particle. The amplitude and extent of these variations change with the viewing geometry, the thermal properties of the particle, and its spin rate and orientation. The typical effects of these parameters on the longitudinal variations are emphasized here. 

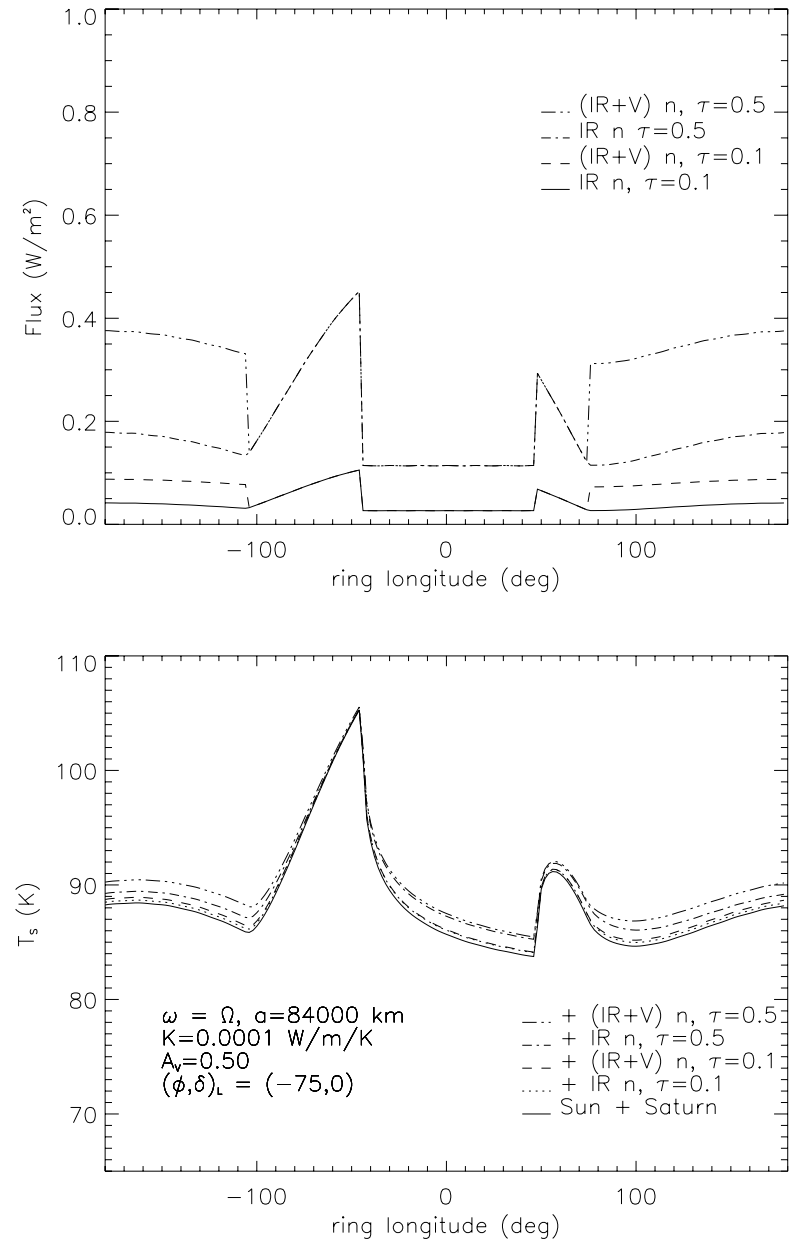

Fig. 7. (Top) Absorbed flux coming from nearby particles on a particle facet at local coordinates $(\alpha, \delta)_{\mathrm{L}}=\left(-75^{\circ}, 0^{\circ}\right)$ as a function of ring longitude $\phi_{\mathrm{R}}$. Infrared absorbed flux (IR $\mathrm{n}$ ), alone or together with absorbed visible flux (IR+V n) is calculated as a function of the optical depth $\tau$ of the ring. The absorbed solar flux is $8 \mathrm{~W} \mathrm{~m}^{-2}$. (Bottom) Corresponding surface temperature when these fluxes are added to the solar and Saturn sources (see Fig. 4 for model parameters).

The large range of possible values for each parameter makes an exhaustive study hopeless. A more detailed description will be done in the future, while comparing the model with specific data sets. It is also shown that this simple thermal model is able to reproduce the observed variation of Saturn's A and $\mathrm{C}$ rings brightness temperatures with solar elevation $B^{\prime}$ (Froidevaux 1981) with simple assumptions on the different model parameters.

\subsection{Influence of the observer's range}

The effective temperature is changing with the local position of the observer as the thermal emission of particles is not isotropic in general. As the observer approaches the rings at constant ring longitude $\phi_{\mathrm{SC}}=180^{\circ}$ and latitude $B$, the local emission angle $\theta_{V}$ varies significantly when the particle moves along its orbit. Large azimuthal variations in effective temperature are thus observed (Fig. 9). Our example illustrates the case for a slowly rotating particle with spin rate $\omega=0.3 \Omega$. As the observer

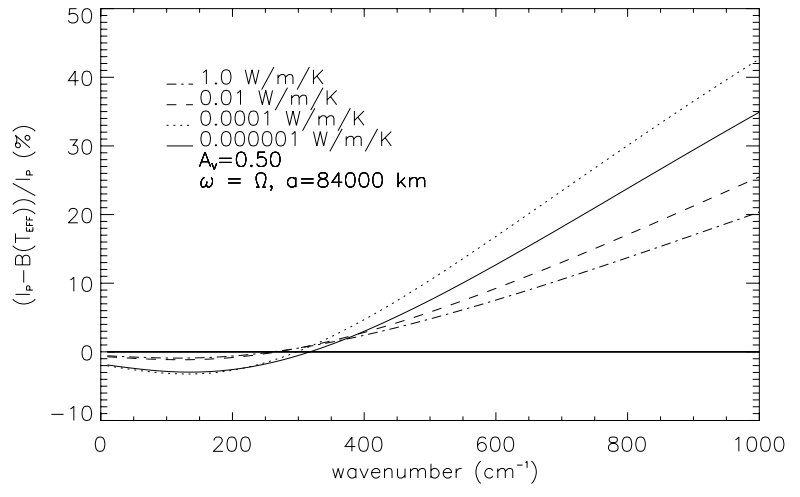

Fig. 8. Residuals between the observed spectrum $I_{\mathrm{P} v}$ and the blackbody emission at temperature $T_{\mathrm{EFF}}$ as a function of thermal conductivity for a synchronous particle with spin axis perpendicular to the ring plane.

approaches the ring (Fig. 9a) near $2.5 r_{\mathrm{S}}$, the effective temperature significantly changes on the West ansa $\left(-180<\phi_{\mathrm{R}}<\right.$ -90). When close enough, the observer no longer observes the sub-solar point but the eastern morning facets of the particle surface that have been in the night for a long time. The visible facets are cooler. After the West ansa, the warmer subsolar facets reappear, and the effective temperature again increases to reach its maximum at $\phi_{\mathrm{R}} \sim 0^{\circ}$. A small decrease happens on the East ansa as the observer sees a little more of the evening facets of the particle. The maximum of the induced variation is about $5 \mathrm{~K}$ on the West ansa for the given parameters. It decreases with increasing $r_{\mathrm{SC}}$ to $\sim 1 \mathrm{~K}$ at $10 r_{\mathrm{S}}$, and is negligible beyond $30 r_{\mathrm{S}}$. When the particle is still closer to the observer, at $a=128000 \mathrm{~km}$, the effect is accentuated as the observer sees more of the cold morning hemisphere. The thermal gradient is as large as $11 \mathrm{~K}$ on the West ansa and about $6 \mathrm{~K}$ just before $\phi_{\mathrm{R}}=180^{\circ}$ on the East ansa (Fig. 9b). As the particle spins faster, the day-to-night temperature constrasts are smaller and the amplitude of the effect decreases.

As expected, the azimuthal variation is reversed relative to $\phi_{\mathrm{R}}=0^{\circ}$ for retrograde spins perpendicular to the ring plane (Fig. 9c). When the observer looks toward the east ansa, the morning side of particles is observed, and the azimuthal variation is the largest. When he looks at the West ansa, he is observing the evening side of particles in this case, cooler than the sub-solar point due to low thermal inertia, but still warmer than the morning side. On a particle with the South pole constantly directed towards the Sun, facets at the same latitude have the same temperatures, and the observer is seeing facets with same thermal histories when he looks at $\pm \phi_{\mathrm{R}}$. The effective temperature decreases toward the ansa as the fraction of the surface in "eternal night" is the largest. This effect should be important in principle for spacecraft observations close to the rings. In the next section, a distant viewpoint is adopted to separate this effect from the others.

\subsection{Influence of the planet}

If the ring sample were a flat surface, Saturn's infrared contribution would be constant with ring longitude $\phi_{\mathrm{R}}$ and would 

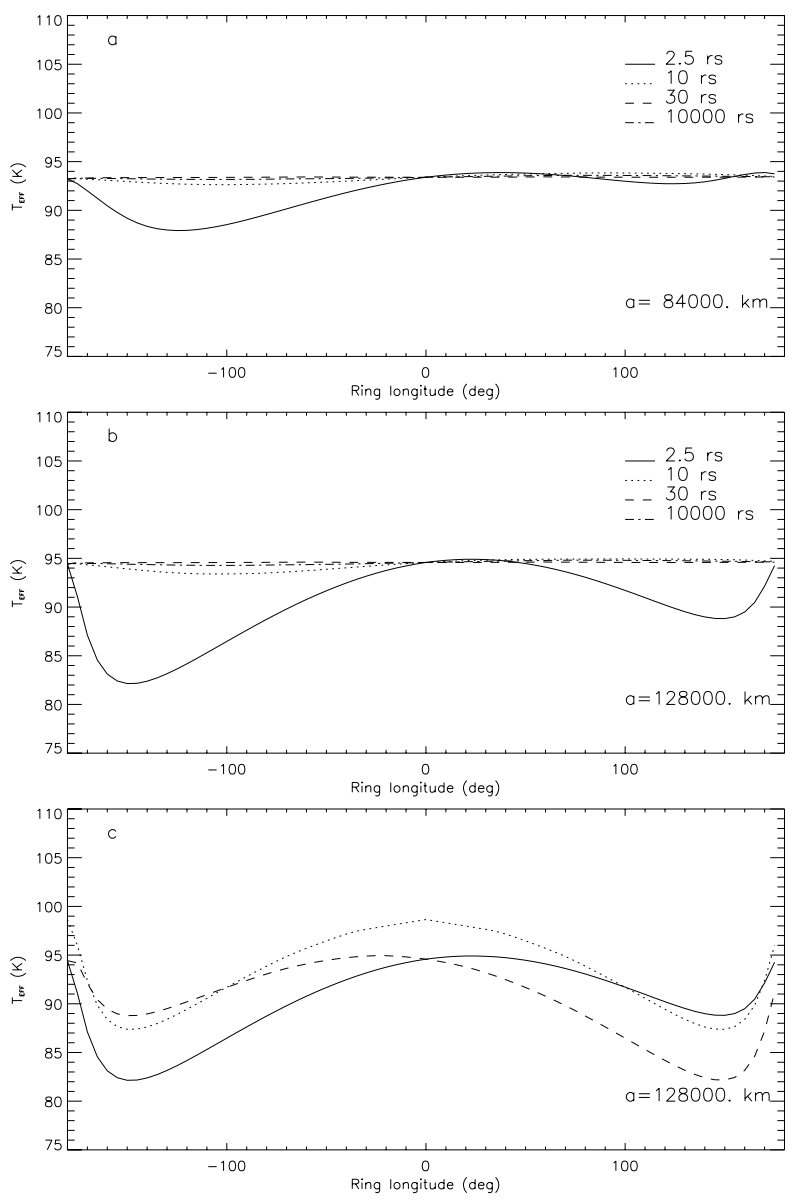

Fig. 9. Azimuthal variation of the particle effective temperature due to a change in the observer range $r_{\mathrm{SC}}$ (given in unit of planet radius $r_{\mathrm{s}}=$ $60330 \mathrm{~km}$ ). The ring particle properties are $K=10^{-4} \mathrm{~W} \mathrm{~m}^{-1} \mathrm{~K}^{-1}$, $A_{\mathrm{V}}=0.5$, and $\omega=0.3 \Omega$. The observer is at elevation $B=0$, and ring longitude $\phi_{\mathrm{SC}}=180^{\circ}$. The Sun is the only heating source. a) The spin axis is perpendicular to the ring plane, $a=84000 \mathrm{~km}$ and $2.5 r_{\mathrm{s}}<$ $r_{\mathrm{SC}}<10000 r_{\mathrm{s}}$. b) Same for $a=128000 \mathrm{~km}$. c) $a=128000 \mathrm{~km}$, the spin axis varies: (full line) perpendicular to the ring plane; prograde (dotted line) parallel to the ring plane, south pole oriented to the Sun direction; (dashed line) perpendicular to the ring plane, retrograde.

not create any azimuthal asymmetry. In the current model, the ring sample is a spherical particle. As the particle is spinning, the infrared flux on each facet varies along the orbit. The very specific position of the planet at the center of the orbit, together with the volumic dimension of the particle, are thus responsible for large azimuthal asymmetries in the effective temperature of the observed hemisphere of particles, wherever the observer is (Fig. 10).

When the observer is at zero phase angle, far away from the planet, the day hemisphere of the particle is observed (Fig. 10, top). The particle's visible face at $\phi_{\mathrm{R}}=-180^{\circ}$ is lit only by the Sun and the neighbors whereas its invisible face is warmed up by both the visible and infrared Saturn contributions and the neighbors. As the particle goes West $\left(\phi_{R}=-90^{\circ}\right)$, the morning hemisphere starts receiving the Saturn contributions and warms up. As it becomes visible there, the effective temperature increases. At $\phi_{\mathrm{R}}=0^{\circ}$, without the planetary shadow crossing event, the visible hemisphere receives contributions
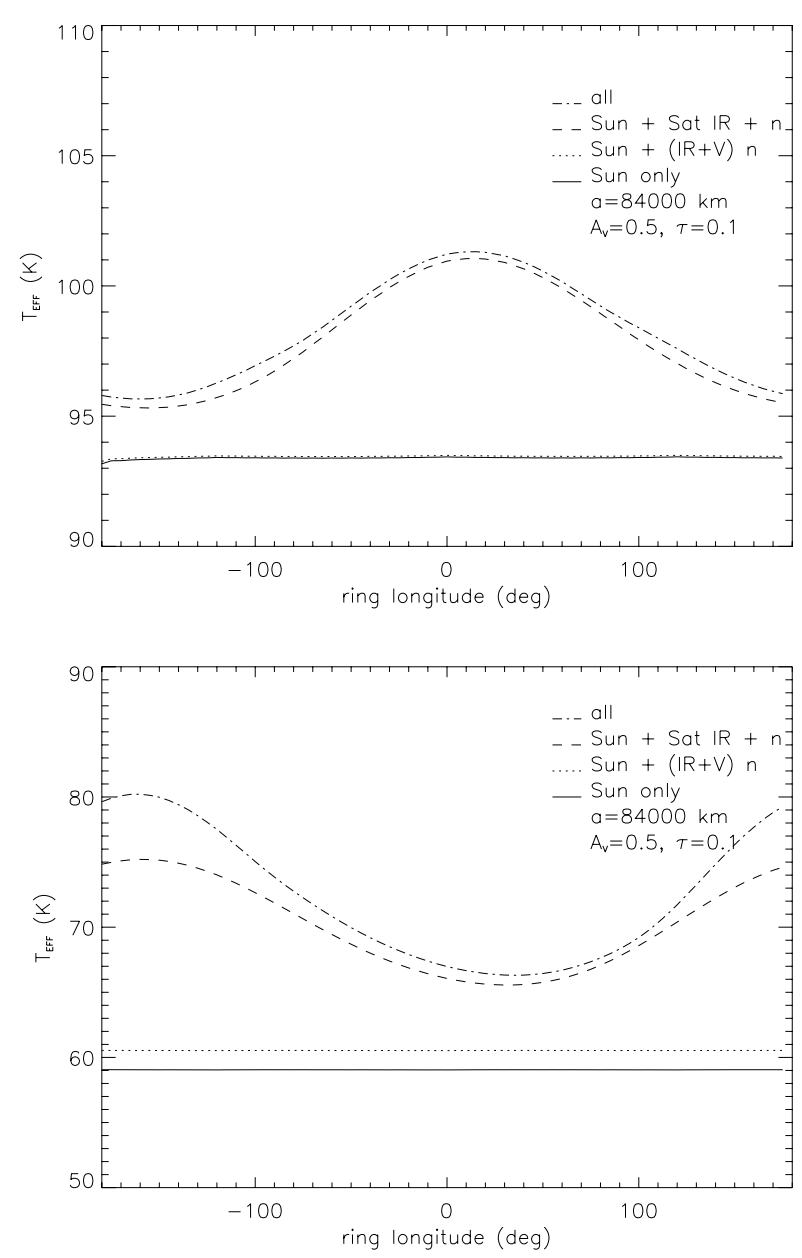

Fig. 10. Azimuthal variation of the particle effective temperature due to Saturn infrared and visible contributions. The ring particle thermal conductivity is $K=10^{-4} \mathrm{~W} \mathrm{~m}^{-1} \mathrm{~K}^{-1}$, its Bond albedo $A_{\mathrm{V}}=0.5$, its spin rate is $\omega=0.3 \Omega$, its direction is perpendicular to the ring plane, the spin is prograde. Particle in the C ring at $a=84000 \mathrm{~km}$, the optical depth is $\tau=0.1$. The observer is at elevation $B=0^{\circ}$, its distance to the planet center is $10000 r_{S}$. The solar elevation $B^{\prime}=0^{\circ}$. The mutual interparticle shadowing and the planetary shadow episode are nto taken into account. (Top) Ring longitude of the observer is $\phi_{\mathrm{SC}}=$ $180^{\circ}$, he is looking at the particle day side. (Bottom) Ring longitude of the observer is $\phi_{\mathrm{SC}}=0^{\circ}$, he is looking at particle night side.

from Saturn and the Sun at the same time and reaches its hottest point. On the East ansa, the evening hemisphere of the particle is lit by Saturn's infrared flux. The hottest point of the visible face decreases as the Sun is the main heating source. The effective temperature comes back to its minimum value. For non-zero thermal inertia, the maximum is shifted towards positive ring longitudes, as the particle hottest point is shifted toward the afternoon hemisphere. Note that the contribution of the Saturn lit hemisphere is detectable in this case as a nearly constant contribution along the orbit. This is due to the fact that the hemisphere lit by this source can hardly be seen except when the particle is about to pass the West ansa or has just passed the East ansa.

When the observer is at a high phase angle (Fig. 10, bottom), the azimuthal variation of effective temperature is opposite to the previous one. When the particle is at $\phi_{\mathrm{R}}=180^{\circ}$, the 

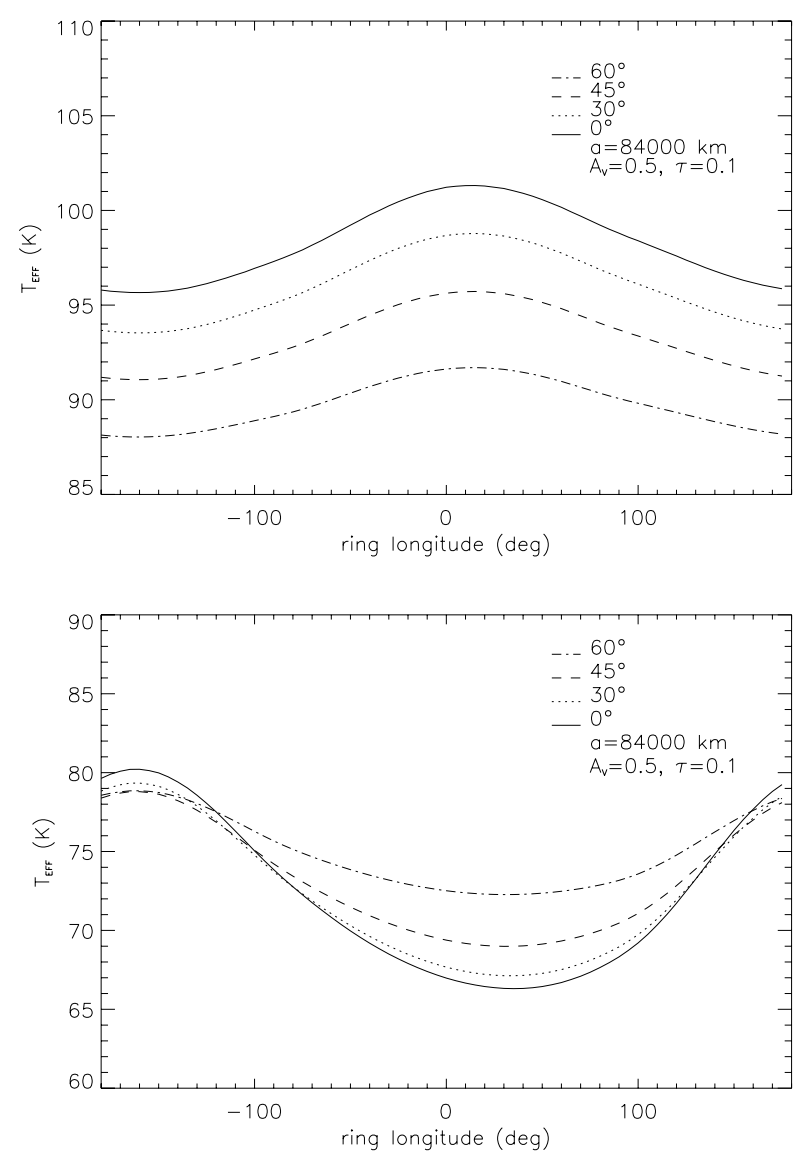

Fig. 11. Azimuthal variation of the particle's effective temperature, versus observer elevation $B$, for two azimuthal positions of the observer. Model parameters are the same as in Fig. 10. The observer elevation varies between 0 and $60^{\circ}$ above the ring plane. (Top) The observer's longitude is $\phi_{\mathrm{R}}=180^{\circ}$, and the visible hemisphere is the particle's day side. (Bottom) The observer's longitude is $\phi_{R}=0^{\circ}$, and the visible hemisphere is the particle's night side.

observer is looking at the night side of the particle that is facing Saturn. Saturn's visible contribution is maximum there, as expected. As the particle goes West, the night face is only partially lit on the morning side, mainly by the infrared Saturn. The effective temperature decreases towards a minimum at $\phi_{\mathrm{R}}=0^{\circ}$ where the night side is facing only deep space and neighbors. When the particle reaches the East ansa $\left(\phi_{\mathrm{R}}=90^{\circ}\right)$, the evening hemisphere is again heated by Saturn and the effective temperature increases again.

As the observer's elevation increases above the ring plane at constant distance and low phase angle $\phi_{\mathrm{R}}=0^{\circ}$ (Fig. 11, top), the effective temperature decreases as the cooler facets of the northern hemisphere are observed. The tendency is reversed for an observer at a high phase angle (Fig. 11, bottom). When in the ring plane, the observer is looking at the night side of particle. As $B$ increases, the warmer lit facets get more and more visible and the temperature increases at almost every longitude, except around $\phi_{\mathrm{R}}=180^{\circ}$ where it stays about constant.
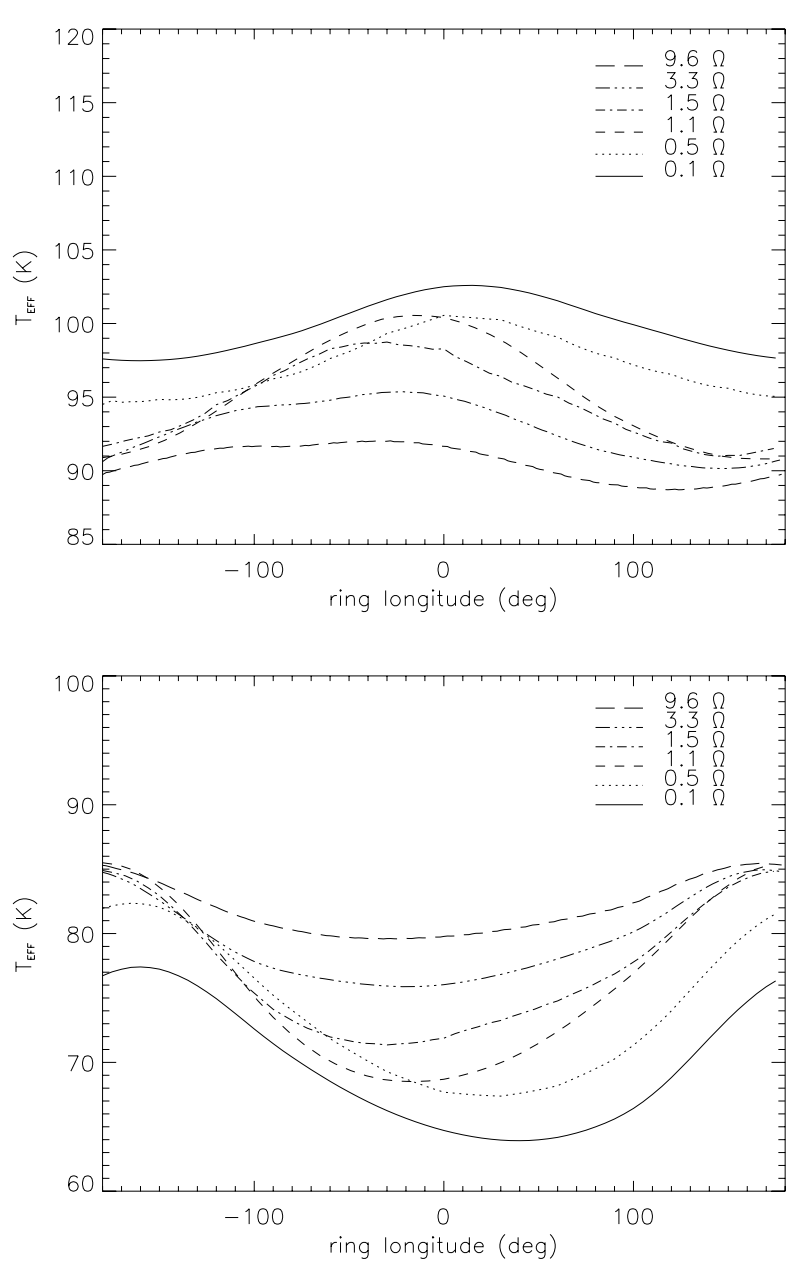

Fig. 12. Azimuthal variation of the particle effective temperature versus particle spin rate $\omega$ for two observer positions. Model parameters are the same as in Fig. 10. All heating sources have been included. Mutual shadowing and eclipse into the planetary shadow are not taken into account. (Top) Longitude of the observer is $\phi_{\mathrm{R}}=180^{\circ}$, so he is looking at the particle day side. (Bottom) Longitude of the observer is $\phi_{\mathrm{R}}=0^{\circ}$, so he is looking at particle night side.

\subsection{Influence of the spin rate}

The azimuthal variation of effective temperature created by Saturn varies in amplitude and shape with particle spin rate (Fig. 12). As the particle spins faster, the thermal history of facets is more uniform, as close facets tend to see the same heating sources at nearly the same time. Their temperature tends to be the same, whatever the observer longitude is. The amplitude of the azimuthal variation decreases. The fastest particles appear to be the coolest when the observer is at a small phase angle or the warmest when the observer is at a high phase angle.

At small phase angle, the temperature peaks just after $\phi_{\mathrm{R}}=$ $0^{\circ}$ (Fig. 12, top) as the heating contributions from Saturn and the Sun overlap on the visible hemisphere. If the particle is slowly spinning, the Saturn's infrared flux hits a visible face that has been under sunlight for a long time. The visible hemisphere is the hottest here, so the small longitudinal shift is due to thermal inertia. When the particle spins faster $(\omega>\Omega)$, the hottest point on the particle has faced Saturn, the Sun and deep 
space, too; it is then cooler than for a slowly spinning particle. If $\omega>2 \Omega$, the particle has made one rotation before arriving at $\phi_{\mathrm{R}}=0^{\circ}$ and the hottest point will appear on the visible face before this point. This explains why the maximum effective temperature is decreasing with increasing spin rate and the peak longitude moving westwards.

At high phase angle (Fig. 12, bottom), the temperature is at its minimum for slow rotators between $\phi_{\mathrm{R}}=0^{\circ}$ and East ansa $\left(\phi_{\mathrm{R}}=90^{\circ}\right)$, as the observer is looking at facets that have usually seen neither the Sun nor Saturn for some time. They have been radiating to deep space, at least for half an orbit. The effective temperature peaks at $\phi_{\mathrm{R}}=180^{\circ}$, when the particle visible hemisphere faces Saturn lit hemisphere. As the particle spins up, its warms up as the visible hemisphere have seen more flux in its thermal history. The minimum shifts toward $\phi_{R}=0^{\circ}$. Here the visible hemisphere has been radiating to deep space recently or has not seen the Sun nor Saturn for the longest period of time. For $\omega=9.6 \Omega$, the effective temperature at high phase angle is not yet equal to the one observed at small phase angle, but is getting close, within $5 \mathrm{~K}$ or $6 \%$.

\subsection{Crossing the planetary shadow}

The crossing of the planetary shadow creates a large azimuthal asymmetry at the place of the shadow and on the East ansa. Figure 13 compares the thermal history of a particle with and without accounting for the shadow crossing for different spin rates and observer positions. First, for a given thermal conductivity, the average effective temperature decreases by a few degrees, whatever the spin rate and the viewing point are, as expected. Second, this event strongly affects the effective temperature around $\phi_{\mathrm{R}}=0^{\circ}$ so that the temperature peak expected at a low phase angle (Fig. 13, top) disappears quite completely. Nonetheless, because thermal inertia is low here, the bump in effective temperature due to Saturn's influence is still observable between $\phi_{\mathrm{R}}=-180^{\circ}$, and the entry into the shadow $\left(\phi_{\mathrm{R}}=-50^{\circ}\right)$.

It is important to note here that at a high phase angle (Fig. 13, bottom), only the effective temperature of fast rotating particles is influenced by the shadow event, as the facets that have suffered the cooling event behind the planet become visible to the observer on the night side very soon after. The effect of the shadow crossing persists there quite imperceptibly for slow and synchronously rotating particles in this viewing geometry. This is therefore a remarkable geometry to put make the presence of a significant fraction of fast rotating particles evident.

\subsection{Prograde and retrograde particles}

Changing the direction of rotation causes large modifications both in the shape of the azimuthal asymmetry and in the average effective temperature of particles at almost all viewing geometries. This is illustrated in Fig. 14 for the case of a particle spinning at rate $\omega=\Omega$ in the prograde or retrograde direction, with a spin perpendicular to the ring plane. Located on the East ansa $\left(\phi_{\mathrm{SC}}=90^{\circ}\right)$, the observer is looking either at the
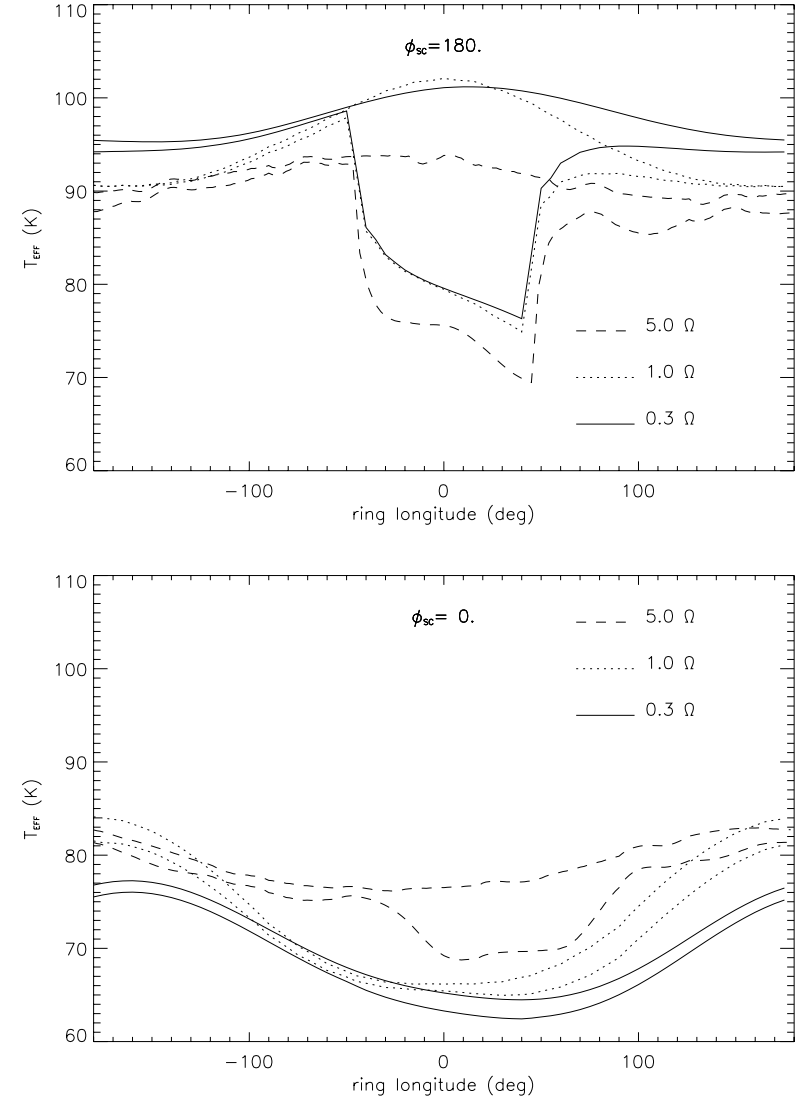

Fig. 13. Crossing the planetary shadow; azimuthal variation of the particle effective temperature versus particle spin rate for four observer positions. Simulation conditions are the same as in Fig. 10. For each spin rate, the top line corresponds to the effective temperature without a shadow crossing event, and the bottom line to the effective temperature with a shadow crossing event. (Top) Longitude of the observer is $\phi_{\mathrm{R}}=180^{\circ}$, so he is looking at the particle day side. (Bottom) Longitude of the observer is $\phi_{\mathrm{R}}=0^{\circ}$, so he is looking at particle night side.

evening side of the retrograde particle or at the morning side of the prograde one. The first one appears much hotter than the second, as expected. On the contrary the prograde particle appears hotter, as seen from the West ansa $\left(\phi_{\mathrm{SC}}=-90^{\circ}\right)$ as its evening side is visible. Differences at intermediate phase angles are more like a change in the mean effective temperature.

Large differences in the azimuthal asymmetries are visible at low and high phase angles between both directions of gyration. At low phase angle $\left(\phi_{\mathrm{SC}}=180^{\circ}\right)$, the bright peak that can be observed just before eclipse $\left(\phi_{\mathrm{R}}=-50^{\circ}\right)$ for a prograde particle disappears. This is due to the fact that for a retrograde particle, the visible face lit there by the Sun has been facing deep space recently instead of the bright infrared source of Saturn. At a high phase angle $\left(\phi_{\mathrm{SC}}=0^{\circ}\right)$, the visible face of the prograde particle at $\phi_{\mathrm{R}}=180^{\circ}$ is facing Saturn along its whole orbit. It is then much hotter than the visible face at $\phi_{\mathrm{R}}=0^{\circ}$, which has seen the Sun about half an orbit sooner, and has been radiateding into space since. The visible face of the retrograde particle at $\phi_{\mathrm{R}}=180^{\circ}$, instead, has been facing deep space for a quarter of orbit, and its visible face at $\phi_{\mathrm{R}}=0^{\circ}$ 

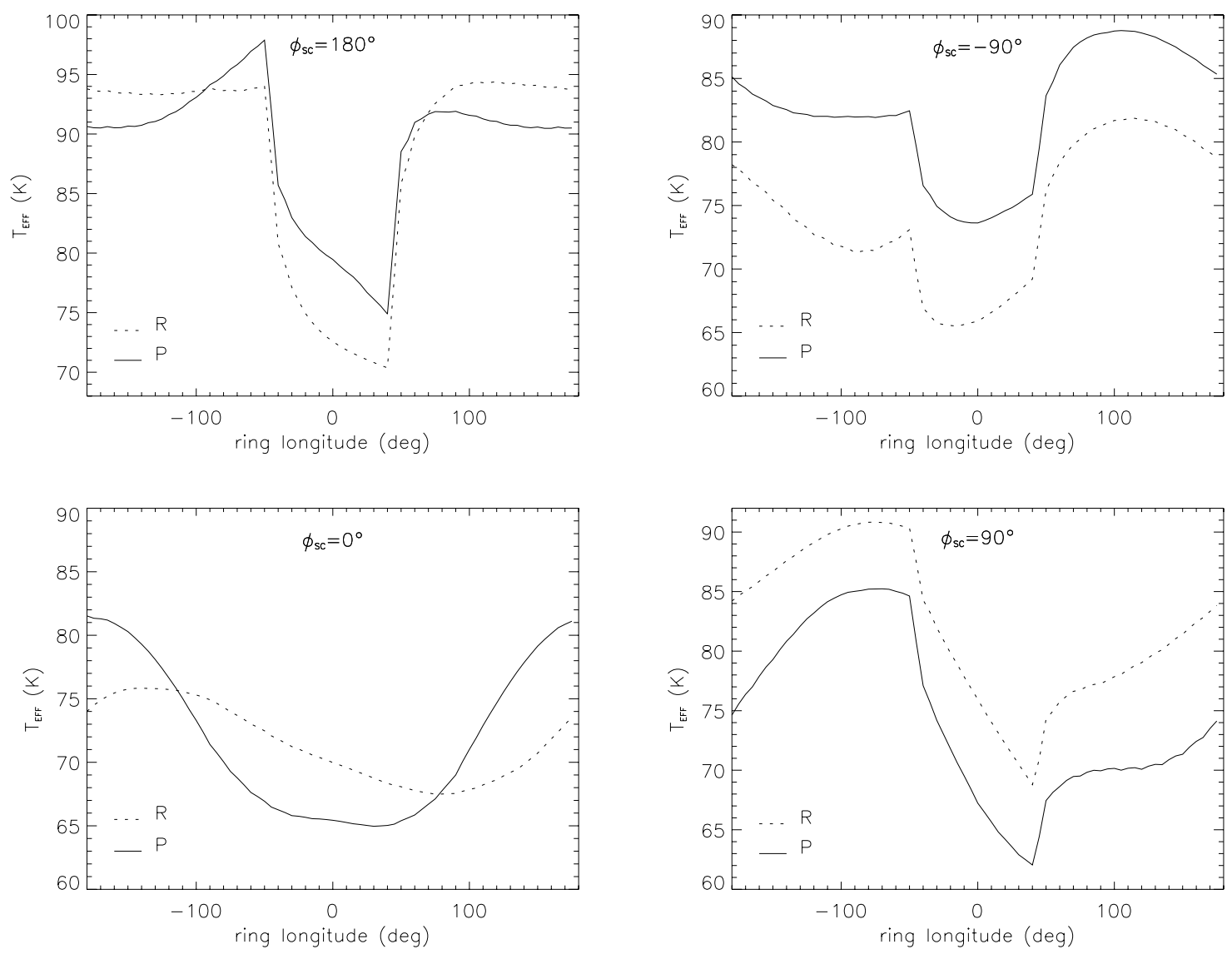

Fig. 14. Influence of the direction of rotation in the azimuthal variation on the particle effective temperature versus observer positions. The particle spin is $\omega=\Omega$ and perpendicular to the ring plane $\left(\alpha_{\mathrm{P}}, \delta_{\mathrm{P}}\right)=\left(0^{\circ}, 90^{\circ}\right)$. Simulation conditions are the same as for Fig. 10 , and the planetary shadow crossing has been taken into account. R: retrograde rotation. P: prograde rotation.

has been radiating into deep space for about half an orbit. This explains the relatively less constrasted azimuthal variations of the retrograde particle from this viewing point. For faster or slower rotators, changes are similar but are more pronounced at intermediate phase angles $\left(\phi_{\mathrm{SC}}=90^{\circ}\right)$ than at low and high phase angles.

\subsection{Influence of the thermal conductivity}

Previous simulations have been made assuming a thermal conductivity $K=10^{-4} \mathrm{~W} \mathrm{~m}^{-1} \mathrm{~K}^{-1}$, which is the order of magnitude estimated for Saturn's rings (Ferrari et al. 2005). Whatever the viewing geometries and the spin rates are, an increasing thermal conductivity has the effect of erasing azimuthal variations, as illustrated in Fig. 15 for a slowly rotating particle with spin axis perpendicular to the ring plane. Those thermal gradients that are about $15 \mathrm{~K}$ for $K \sim 10^{-4} \mathrm{~W} \mathrm{~m}^{-1} \mathrm{~K}^{-1}$ are reduced to only a few Kelvin for $\mathrm{K}$ larger than $10^{-2} \mathrm{~W} \mathrm{~m}^{-1} \mathrm{~K}^{-1}$. For very large $\mathrm{K}$, the effective temperature tends to be constant along the azimuth, with the same value at any observer positions, which is expected as $B \sim B^{\prime}$. The temperature contrast between the shadow area $\left(\phi_{\mathrm{R}}=0^{\circ}\right)$ and the West ansa $\left(\phi_{\mathrm{R}}=-90^{\circ}\right)$ is the best location to look at to estimate the thermal conductivity $K$.

\subsection{Dispersion in spin rates and obliquities}

The ring population is expected to have dispersed distributions of spin rates and obliquities. Figure 16 shows how the effective temperature of a prograde particle changes its spin obliquity $\left(\alpha_{\mathrm{P}}, \delta_{\mathrm{P}}\right)$, both angles being uniformly dispersed in the range $\left[0^{\circ}, 180^{\circ}\right]$ by steps of $30^{\circ}$. A change in right ascension $\alpha_{\mathrm{P}}$ of the spin orientation does not significantly affect the overall shape of the azimuthal variations of the effective temperature generated by Saturn. In most geometries, it is equivalent to a change in Bond albedo.

The largest variations induced by a variation of $\delta_{\mathrm{P}}$ in the plane perpendicular to the Saturn-Sun direction are observed either from the East or the West ansae, whatever the spin rate is. For example, when the observer is located at longitude $\phi_{\mathrm{SC}}=90^{\circ}$ (East ansa), the visible face of the rolling particle $\left(\alpha_{\mathrm{P}}, \delta_{\mathrm{P}}\right)=\left(0^{\circ}, 0^{\circ}\right)$ is the North pole or the morning side for the perpendicular spin $\left(\alpha_{\mathrm{P}}, \delta_{\mathrm{P}}\right)=\left(0^{\circ}, 90^{\circ}\right)$. The main difference in thermal histories happens after the West ansa at $\phi_{\mathrm{R}}=-90^{\circ}$, where the North pole of the rolling particle accumulates incoming flux on a hotter surface than the morning face of the particle with spin perpendicular to the ring plane. It makes the average effective temperature of the rolling particle the largest for this viewing point. The tendency is opposite when viewing the ring from the West ansa. 

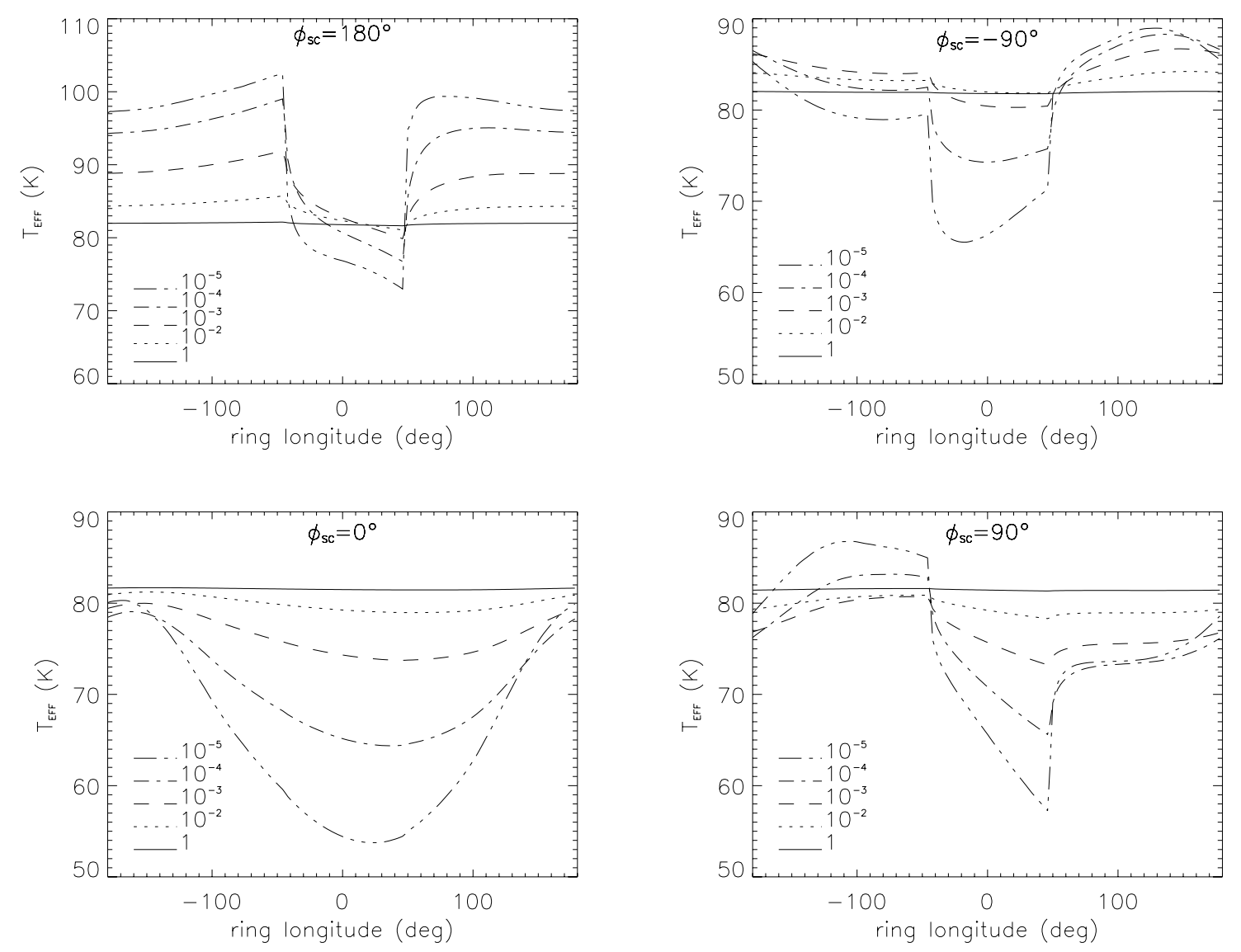

Fig. 15. Influence of the thermal conductivity $K\left(\mathrm{~W} \mathrm{~m}^{-1} \mathrm{~K}^{-1}\right)$ on the azimuthal variation of the particle effective temperature for four observer positions. The spin direction is perpendicular to the ring plane, the spin is prograde, and $\omega=0.3 \Omega$. The other simulation conditions are similar to those of Fig. 10 with the planetary shadow episode included.

Most viewing geometries are also sensitive to a variation in $\delta_{\mathrm{P}}$ on a plane parallel to the Sun-Saturn direction and perpendicular to the ring plane, whatever the spin rate is. The largest dispersions in effective temperature are observed at high and low-phase angles viewing points. At zero phase angle $\left(\phi_{\mathrm{SC}}=180^{\circ}\right)$, the rolling particle $\left(\alpha_{\mathrm{P}}, \delta_{\mathrm{P}}\right)=\left(270^{\circ}, 0^{\circ}\right)$ is the warmest because the visible face is the South pole, which is lit by the Sun on most of the orbital revolution. Of course, this particle is the coolest for an observer at high phase angle $\left(\phi_{\mathrm{SC}}=0^{\circ}\right)$, as the visible face is the North pole which is only heated by Saturn during a orbital revolution.

The overall dispersion of $T_{\mathrm{EFF}}$ induced by this distribution of spin obliquities is about $10 \mathrm{~K}$ for the given particle properties (Fig. 16). The average effective temperature of the population is $T_{\mathrm{EFF}, \mathrm{P}}=\left(1 / N \sum_{\left(\alpha_{\mathrm{p}}, \delta_{\mathrm{P}}\right)} T_{\mathrm{EFF}}^{4}\right)^{1 / 4}$, assuming that obliquities are equiprobable. Remarkably, large azimuthal asymmetries induced by Saturn onto the spherical particles are conserved despite averaging over a wide range of obliquities.

The average effective temperature $T_{\mathrm{EFF}, \mathrm{P}}$ of a population of randomly oriented spins also varies with spin rate (Fig. 17). When observed at low phase angle, the slowest particles are the hottest, as their visible hemisphere is always lit by the Sun or Saturn. As particles spin faster, the average effective temperature decreases, and the overall shape of the azimuthal asymmetries evolves (Fig. 17, left). At the exit of the shadow $\left(\phi_{\mathrm{R}}>50^{\circ}\right)$, fast rotating particles are warming up more progressively than slower ones, as a similar incoming flux is relatively more widerly distributed on the surface of the particle. This is also the case on the West ansa $\left(\phi_{\mathrm{R}} \sim-90^{\circ}\right)$, where the peak before entry into the shadow $\left(\phi_{\mathrm{R}} \sim-50^{\circ}\right)$ is more contrasted for slowly rotating particles.

If the distribution of spins is uniformly distributed, the azimuthal variation of $T_{\mathrm{EFF}, \mathrm{P}}$, averaged over spins and obliquities, is very similar to that of the hottest particles for this viewing angle, i.e. the slowest ones (Fig. 17, right). This case can be considered equally as an equilibrate mixture of slowly rotating particles with $\omega \leq \Omega$ (population 1) and as fast rotators with $\omega>\Omega$ (population 2) of contributive fraction $f=0.5$ to the total emitted flux. A ring made of population 2 only $(f=1)$ would display a very different behavior than a ring made of population 1 ( $f=0$ ) (Fig. 17, right) for the reasons given above. The average effective temperature for a ring population with a Maxwellian distribution of spin rates like $\omega \mathrm{e}^{-\omega}$ (Richardson 1994) is close to the uniform distribution case as this distribution peaks at $\omega=\Omega$, one among the hottest particles of the population. But it appears much flatter at the exit of the shadow $\left(\phi_{R}>50^{\circ}\right)$. The observed effective temperature of a population of particles with randomly distributed spin rates and obliquities still depends on the actual laws of probability for these distributions. Numerical simulations of the local 

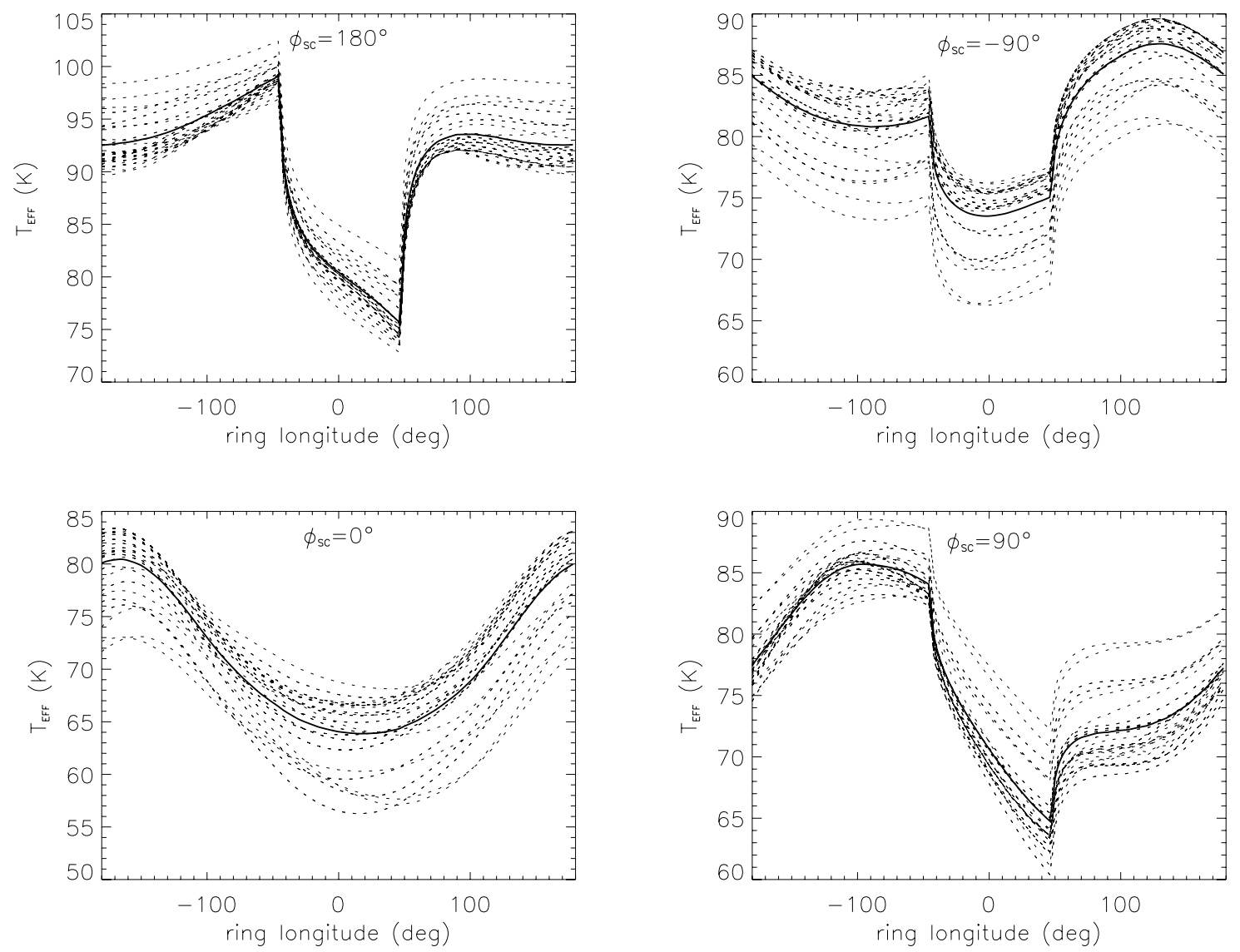

Fig. 16. Influence of the spin obliquity on the azimuthal variation of the particle effective temperature (dotted lines). The effective temperature is displayed here for $N=49$ different obliquities $\left(\alpha_{\mathrm{P}}, \delta_{\mathrm{P}}\right)$ uniformly distributed in the range $\left[0^{\circ}-180^{\circ}\right]$ by steps of $30^{\circ}$. The thick full line is the average effective temperature of a ring population that would have such a distribution in spin obliquities. The particles are prograde and $\omega=\Omega$. Other simulation conditions are the same as used in the case of Fig. 10, and the planetary shadow crossing is included.
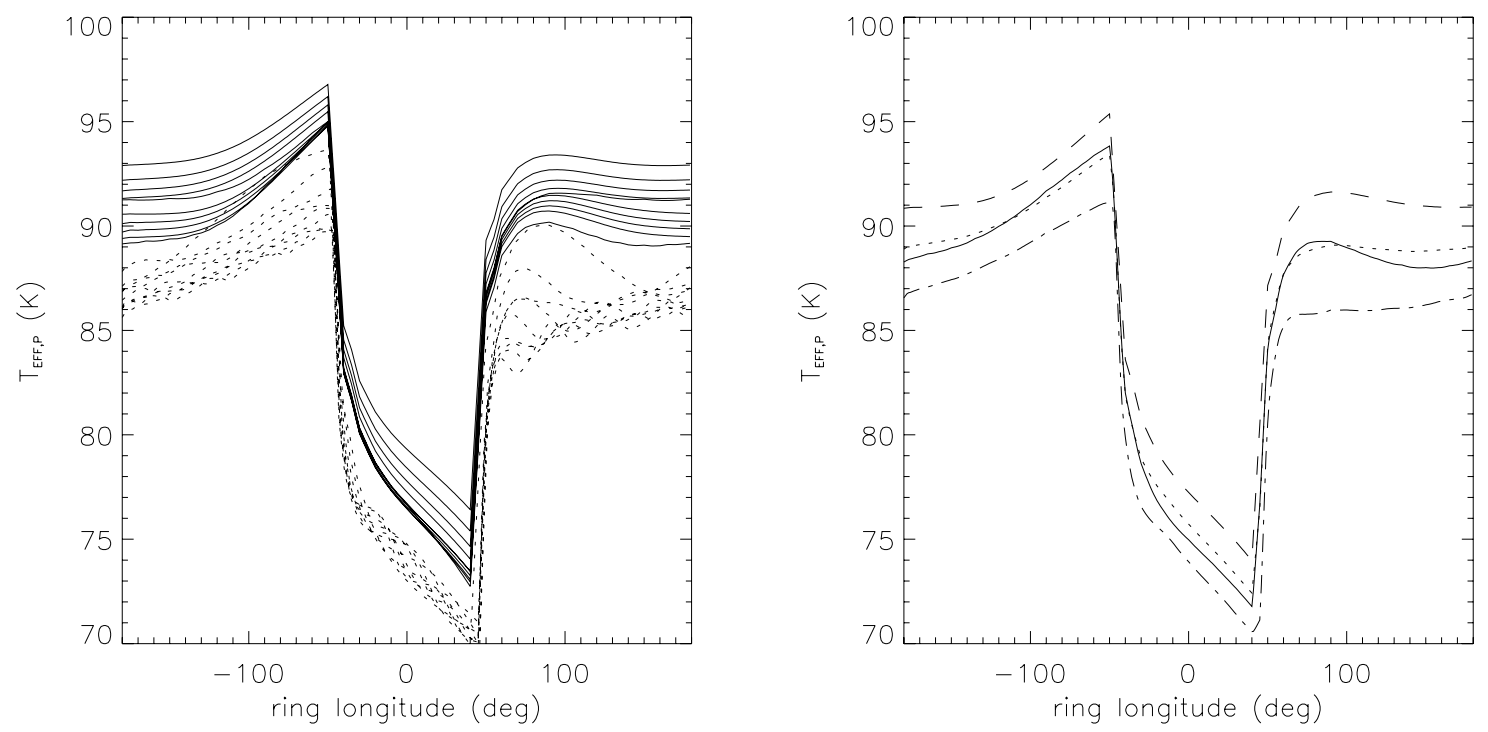

Fig. 17. Influence of a dispersion in spin rates on the average effective temperature $T_{\mathrm{EFF}, \mathrm{P}}$ of the ring population. Spins are prograde and obliquities are equiprobable (cf. Fig. 14). Simulation conditions are the same as in Fig. 14. The observer position is located at longitude $\phi_{\mathrm{SC}}=$ $180^{\circ}$. (Left) Average effective temperatures for a uniform distribution of obliquities as a function of the spin rate for: $\omega \leq \Omega$ (full line, population 1 , in the range $[0.1 \Omega-1 \Omega]$ by steps of $0.1 \Omega$ ) and for $\omega>\Omega$ (dotted line, population 2 , in the range $[1 \Omega-10 \Omega]$ by steps of $1 \Omega$ ). (Right) Average effective temperature for a uniform distribution of spin rates (dot), for a uniform distribution of slow spin rates (population 1, dash), for a uniform distribution of fast spin rates (population 2, dot-dash), or a Maxwellian distribution $\omega \mathrm{e}^{-\omega}$ (full), assuming a uniform distribution of spin obliquities. 
ring dynamics can provide more "realistic" distributions of spin rates and obliquities. In near future, they will be introduced in this thermal model (Charnoz et al. 2003). A comparison with the actual thermal emission of rings as a function of ring longitude will then allow us to test if these distributions are actually realistic.

\section{Mutual shadowing and ring brightness temperature}

Beyond the mutual heating between particles, mutual shadowing is another important collective effect on the ring thermal emission. In this standard model, the ring population is assumed to spread in a monolayer. This is reasonable if only the largest ring particles in a ring of moderate optical depth are considered (Brahic \& Sicardy 1981; Salo 1987b; Richardson 1994). Mutual shadowing is the main cause of the tilt effect observed in Saturn's rings in the mid-infrared, i.e. the variation in ring brightness temperature $T_{\mathrm{B}}$ with the solar elevation $B^{\prime}$ (Esposito et al. 1984). As the Sun sets, shadows are longer, mutual shadowing larger, and the particles are cooler. Mutual shadowing between particles has been neglected in previous simulations by fixing the non-shadowing factor $C\left(\tau, B^{\prime}\right)=1$; it is considered here. It only affects the relative amount of incoming solar flux to the total absorbed flux as shown in Eq. (4). Therefore previous studies are not qualitatively altered.

Figures $18 \mathrm{a}$ and $18 \mathrm{~b}$ show that the present model is able to reproduce the tilt effect on Saturn's A and C rings observed from the ground at $20 \mu \mathrm{m}$ wavelength on the West ansa $\left(\phi_{\mathrm{R}}=-90^{\circ}\right)$. Laboratory measurements by Froidevaux (1981) of the mutual shadowing $C\left(\tau, B^{\prime}\right)$ for randomly distributed particles were used here (Fig. 18c). The brightness temperature $T_{\mathrm{B}}$ is calculated at $\lambda_{0}=c / v_{0}=20 \mu \mathrm{m}$ from Eq. (1) and $I_{\mathrm{P} v_{0}}=$ $B_{v_{0}}\left(T_{\mathrm{B}}\right)$, which includes the visibility factor $C(\tau, B)$. The $\mathrm{C}$ ring brightness temperatures can be fitted with very slowly rotating particles of low thermal conductivity $K=0.0001 \mathrm{~W} \mathrm{~m}^{-1} \mathrm{~K}^{-1}$ and Bond albedo $A_{\mathrm{V}}=0.52$ or with fast-rotating particles with lower albedo $A_{\mathrm{V}}=0.32$, both having spin axes perpendicular to the ring plane. Froidevaux (1981) was able to reproduce the C-ring brightness temperature for slowly rotating particles with $f=2$, random shadowing, and somewhat larger Bond albedo, $A_{\mathrm{V}}=0.55$ or with fast rotating particles $A_{\mathrm{V}}<0.35$. For the A ring, the brightness temperature curve can be almost reproduced with slowly rotating particles with $A_{\mathrm{V}}=0.55$ or with fast rotators with $A_{\mathrm{V}}=0.35$. Froidevaux (1981) has fitted the observed behavior with the same albedo for slowly rotating particles $(f=2)$ but not for fast-rotating ones $(f=4)$, as the albedo had to be lower than 0.05 .

Consideration of the Bond albedo values can help in discriminating between slow and fast rotators with ground-based observations of the tilt effect in thermal infrared. More data are needed at different viewing angles and wavelengths to constrain the spin of particles.

\section{Conclusions}

The thermal model presented in this paper provides new insights into what might be the thermal emission of spherical
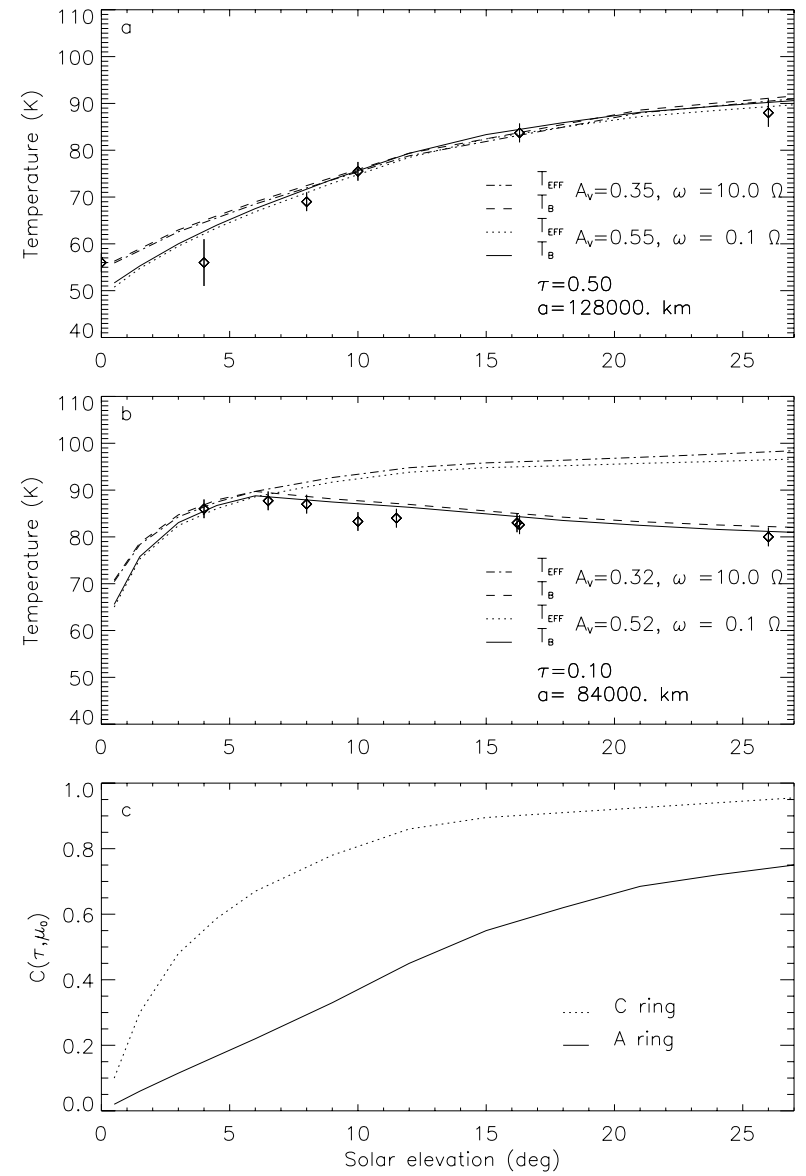

Fig. 18. Particle effective temperatures and ring brightness temperatures on the West ansa of the Saturn' A and C rings versus solar elevation $B^{\prime}$. Data points are ground-based observations obtained at $20 \mu \mathrm{m}$ (Esposito et al. 1984 for a review). Calculations include all heating sources, assuming optical depth $\tau$ at distance a. The thermal conductivity $K$ is fixed at $10^{-4} \mathrm{~W} \mathrm{~m}^{-1} \mathrm{~K}^{-1}$. The particle spin axis is perpendicular to the ring plane and prograde. a) A ring. Good fit is obtained for fast $(\omega=10 \Omega)$ and slow rotating $(\omega=\Omega / 10)$ particles with Bond albedo $A_{\mathrm{V}}=0.35$ or $A_{\mathrm{V}}=0.55$, respectively. b) C ring. Good fit is obtained for fast $(\omega=10 \Omega)$ and slow rotating $(\omega=\Omega / 10)$ particles with Bond albedo $A_{\mathrm{V}}=0.32$ or $A_{\mathrm{V}}=0.52$, respectively. c) Nonshadowing factor $C\left(\tau, B^{\prime}\right)$ in the case of randomly distributed ring particles as measured experimentally by Froidevaux (1981) for A and C rings.

spinning ring particles distributed in a monolayer. The main conclusions are:

- The Saturn infrared emission on a population of spherical particles, dispersed in a monolayer and having both constant and identical spin rate and obliquity, generates large azimuthal variations in temperature.

- Three important factors govern the amplitude and shape of these variations: the particle spin rate, its thermal inertia, and the viewing geometry.

- With increasing spin rate, the amplitude decreases and the longitude of the peak temperature moves around (Fig. 12). The presence of fast-spinning particles in such a ring can be easily detected from a high phase angle viewing point if 
a transient cooling due to the shadow crossing is observed (Fig. 13).

- With increasing thermal inertia (or thermal conductivity), the amplitude decreases, and the cooling and heating regimes at the entry and exit of the planetary shadow almost disappear (Fig. 15).

- The peak temperature of this asymmetry takes place at different longitudes depending mainly on the observer's position. Without the major cooling event into the planetary shadow, this maximum would be located at a longitude opposite to the observer position, i.e. at $\phi_{\mathrm{R}}=\phi_{\mathrm{SC}} \pm 180^{\circ}$ (Figs. 10, 14).

- Azimuthal variations in effective temperature are still present if particle spin rate and obliquities are randomly distributed (Fig. 17). The amplitude and shape of the variations are functions of the law of probability for these distributions.

This model is based on simplifying assumptions. Numerical simulations of the local ring dynamics show that particles of different sizes have different spin rates and obliquities. We recently developed numerical simulations of the local dynamics of ring particles of different sizes (Charnoz et al. 2003). They will provide "realistic" dynamical inputs to this thermal model in near future more, such as the distribution of spins magnitude and obliquities, the vertical structure of the ring layer, and a non-shadowing factor for the typical vertical dynamics of particles at equilibrium. Only heat conduction is considered here. The transport of energy by radiation is not negligible in the far infrared, so its effect on the observed temperature should be studied. This model will be tested in the near future against observations of Saturn's rings provided by the CIRS infrared spectrometer on board the CASSINI spacecraft. It will also be used to analyze infrared images of Saturn's rings obtained from the ground in the mid-infrared with the VISIR/VLT spectroimager. Ground-based and CIRS data remain very complementary, increasing the number of viewing angles and obtaining new constraints on the local dynamical properties of Saturn's main rings.

Acknowledgements. This work was supported by the Programme National de Planétologie and the Centre National d'Etudes Spatiales. We warmly thank Dr. Linda Spilker for continuing discussions on this work and Dr. Heikki Salo for very helpful criticism of this manuscript. We acknowledge Dr. S. Charnoz for discussions on the dynamical backgrounds of this work.

\section{References}

Araki, S. 1991, Icarus, 90, 139

Aumann, H. H., \& Kieffer, H. H. 1973, ApJ, 186, 305

Brahic, A., \& Sicardy, B. 1981, Nature, 289, 447

Clark, R. N., \& McCord, T. B. 1980, Icarus, 43, 16

Charnoz, S., Leyrat, C., Ferrari, C., et al. 2003, BAAS, abs.\# 35/20.01

Cuzzi, J. N., Lissauer, J. J., Esposito, et al. 1984, in Planetary Rings, ed. R. Greenberg, \& A. Brahic (Tucson: The University of Arizona Press), 73

Esposito, L. W., O'Calaghan, M., \& West, R. A. 1983, Icarus, 56, 439

Esposito, L. W., Cuzzi, J. N., Holberg, et al. 1984, in Saturn, ed. T. Gehrels, \& M. S. Matthews (Tucson: The University of Arizona Press), 463

French, R. G., \& Nicholson, P. D. 2000, Icarus, 145, 502

Ferrari, C., Galdemard, P., Lagage, P. O., et al. 1999, BAAS, 31, 1588

Ferrari, C., Galdemard, P., Lagage, P. O., et al. 2005, A\&A, 441, 379

Flasar, M., \& the CIRS Science Investigation Team 2004, Space Sci. Rev., 115, 169

Froidevaux, L., \& Ingersoll, A. P. 1980, J. Geophys. Res., 85, 5929

Froidevaux, L., Matthews, K., \& Neugebauer, G. 1981, Icarus, 46, 18

Froidevaux, L. 1981, Icarus, 46, 4

Kawata, Y. 1983, Icarus, 56, 453

Klinger, J. 1981, Icarus, 47, 320

Pilcher, C. B., Chapman, C. R., Lebofsky, L. A., et al. 1970, Science, 167,1372

Poulet, F., \& Cuzzi, J. N. 2002, Icarus, 160, 350

Puetter, R. C., \& Russel, R. W. 1977, Icarus, 32, 37

Richardson, D. 1994, MNRAS, 269, 493

Rosen, P. A. 1989, Scientific Report No. D845-1989-1, Stanford University

Salo, H. 1987a, Earth, Moon, Planets, 38, 149

Salo, H. 1987b, Icarus, 70, 37

Salo, H. 1991, Icarus, 90, 254

Salo, H. 1995, Icarus, 117, 287

Salo, H., \& Karjalainen, R. 2003, Icarus, 164, 428

Shu, F. H., Cuzzi, J. N., \& Lissauer, J. J. 1982, Icarus, 53, 185

Spilker, L. J., Wallis, B. D., Ferrari, C., et al. 2002, BAAS, 33, 1096

Spilker, L., Ferrari, C., Cuzzi, J. N., et al. 2003, Planet. Space Sci., 51, 929

Spilker, L., Ferrari, C., Pilorz, et al. 2005, Icarus, submitted 